\title{
Evolutionary response of a native butterfly to concurrent plant invasions: Simulation of population dynamics
}

\author{
Manuel García-Quismondo a,b,c,*, J. Michael Reed ${ }^{\text {b }}$, Frances S. Chew ${ }^{\text {b }}$, \\ Miguel A. Martínez-del-Amor ${ }^{a}$, Mario J. Pérez-Jiménez ${ }^{\mathrm{a}}$ \\ ${ }^{a}$ Research Group on Natural Computing, University of Sevilla, ETS Ingeniería Informática, Av. Reina Mercedes, s/n, Sevilla 41012, Spain \\ ${ }^{\mathrm{b}}$ Dept. of Biology, Tufts University, Medford, MA 02155, USA

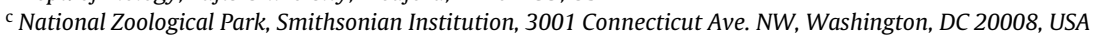

Keywords:

Pieris oleracea

Membrane computing

Population dynamics

Probabilistic Guarded Scripted P system

Invasive species

\begin{abstract}
A B S T R A C T
The habitat of the green-veined white butterfly Pieris oleracea in eastern North America has undergone invasions by the exotic plant garlic mustard (Alliaria petiolata), which is replacing native hosts of $P$. oleracea such as Cardamine diphylla. A. petiolata was originally lethal to most larvae of the native butterfly but during the past $20+$ years it has been incorporated successfully into the larval diet, likely through evolutionary change. The region was also invaded by another exotic plant, Cardamine pratensis, on which the native butterfly larvae readily develops, allowing the possibility of population rescue. Further complicating the butterfly's reproductive dynamics, it is multigenerational within a summer, and host plant availability and location change during the summer. Our goal is to model the expected dynamics of the native butterfly population in this evolving, dynamic landscape by using a new bio-inspired paradigm known as membrane computing.

In this context, a Probabilistic Guarded Scripted P system has been designed to model and explore the conditions under which an allele conferring ability of $P$. oleracea larvae to develop on A. petiolata might have proliferated. The design describes a population dynamics model whose parameter values are derived from experimental and observational data. Our modeling framework is spatially explicit and our model integrates seasonal as well as annual dynamics. The simulation results from our model qualitatively match our field observations and experimental laboratory results, and agree with the results from a previous model on the genotypic adaptation of this butterfly species. From the simulations we identified the likely trajectories for the spatio-temporal distribution of alleles enabling $P$. oleracea to use the invasive plant species across this selective and phenological mosaic.
\end{abstract}

\section{Introduction}

The effects on native herbivorous insects from exotic plant invasions relative to their normal host plants occur at both ecological and evolutionary time scales (Bezemer et al., 2014). These can include "no effect" (e.g. effects of crown vetch Coronilla on the native butterfly Colias philodice (Karowe, 1990)), to positive effects such as increased population sizes and expanded habitat occupancy in native butterflies using non-native species as larval hosts (e.g. Speyeria idalia larvae feeding on non-native Viola bicolor (Shuey et al., 2016) or Euphydryas phaeton using non-native Plantago lance-

\footnotetext{
* Corresponding author at: Research Group on Natural Computing, University of Sevilla, ETS Ingeniería Informática, Av. Reina Mercedes, s/n, Sevilla 41012, Spain. E-mail address: mgarciaquismondo@us.es (M. García-Quismondo).
}

olata (Brown et al., 2017)), possibly altered larval or adult defense against predation (e.g. Anartia jatrophe larvae using non-native $P$. lanceolata) store some of the iridoid glycosides found in this plant (Knerl and Bowers, 2013), or increasing the number of generations per year (voltinism) (e.g. Pieris napi and Battus philenor using non-native hosts (Herlihy et al., 2014; Shapiro, 1975a,b; Sims and Shapiro, 1984)). Of concern, however, is that novel exotic plants may present native herbivorous insects with 'sensory traps' that are attractive to ovipositing adults but poorly support subsequent larval growth and development (Chew et al., 2012; Harvey et al., 2010; Keeler and Chew, 2008; Keeler et al., 2006).

In natural systems native species may often be exposed to closely sequential or concurrent invasion events from a variety of exotic species with contrasting effects on native species. Predicting the trajectory of a native species' response to such concurrent encounters with exotic species is not straightforward. Further con- 


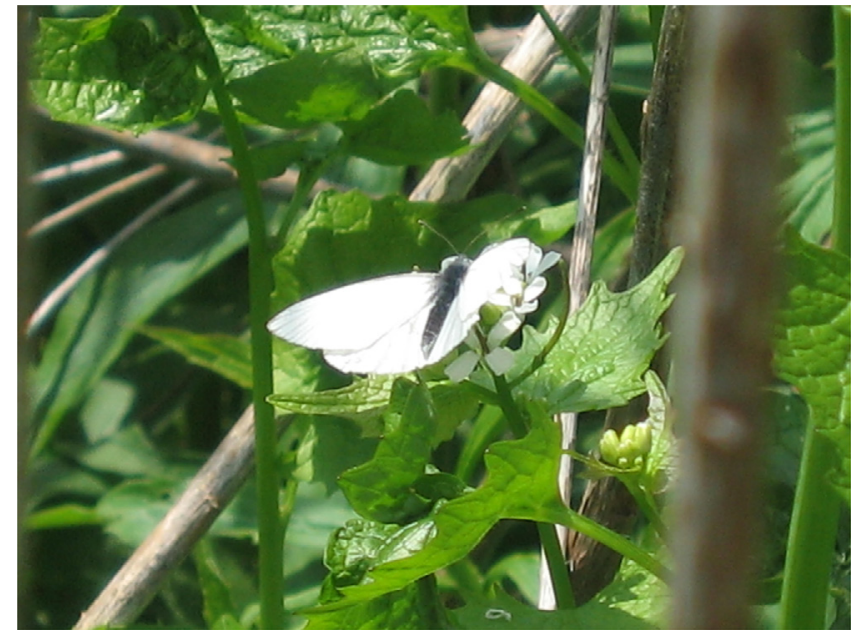

Fig. 1. Adult Pieris oleracea nectaring at flowers of garlic mustard, Alliaria petiolata. Photo by F.S. Chew.

tributing to the complexity of effects from concurrent invasion events, a selective spatial and phenological mosaic may be created by the timing and spatial distribution of exotic species as they spread in a region. The trajectory of a native species' response as it encounters exotic species across this mosaic will depend on the details of genetic variation, natural history, life history, chance, and how different parts of the insect population fare in response to dispersal of individuals that survive selective filters in different parts of the mosaic. Here we model a biological system of a native herbivorous insect whose native host plant is being replaced by an exotic invasive plant that has strongly detrimental effects on the native insect, and a closely sequential or possibly concurrent invasion by a second exotic plant with strongly positive effects.

A recovery of population size at our study area (at the nexus of invasions) has already been documented (Chew et al., 2012; Herlihy et al., 2014), as has apparent adaptation of the native insect population to the formerly detrimental exotic plant, whose bolting (flowering) stage but not the rosette stage is now being incorporated into the native insect's diet (Keeler and Chew, 2008). Alliaria petiolata M. Bieb. (Cavara \& Grande) is present in the system in its rosette stage throughout the entire year, but its bolting stage is present only during the first generation of butterflies each year. Prior stochastic models of this system have explored the competing top-down versus bottom-up influences on population persistence of the native butterfly $P$. oleracea Harris (Lepidoptera: Pieridae) (Keeler et al., 2006) (Fig. 1), and the putative effects of two exotic parasitoid species on population decline and recovery of the butterfly, and an exploration of whether mutation or residual polymorphism is a more probable source for genetic variation (Morton et al., 2015).

Our goals in this model address components that have not yet been modeled, in part because of limitations of the (prior) modeling framework. Specifically, we assess: a) Effects of simultaneous concurrent invasions of two hosts - one "good" and one initially "bad"- for the native butterfly, and their effects on the proliferation of genotypes adapted to the "initially bad" host plant. (b) Effects of spatially contiguous but ecologically distinct habitats with different suites of potential host plants and thus seasonally and spatially distinct selection regimes within a breeding season that includes up to three generations of the native butterfly. (c) Butterfly population dynamics and evolution between generations within a breeding season and from year-to-year, tracking the proliferation of genotypes adapted to the exotic invasive plant that was a formerly poor host.
These explorations permit us to assess the possible contributions of the "good" exotic host and the successful incorporation of the initially "bad" exotic host into the butterfly diet to population recovery at this site. They further allow us to explore the hypothesis that near-concurrent invasion by the "good" exotic host may have contributed to spread of the genetic adaptation to the formerly "bad" exotic host.

\section{Computational modeling frameworks in population dynamics}

Traditionally, the study of complex systems (and population dynamics, in particular) has been addressed by using ordinary differential equations (ODEs). This approach shows in turn some drawbacks when used for discrete and stochastic processes, and poses some inflexibility to changes, that can cause a big impact in the defined equations (Costantino et al., 1997; Grimm, 1999; Grimm et al., 1999; Judson, 1994; Romero-Campero and PérezJiménez, 2008a). Hence, other computational paradigms have been considered to define more accurate models. One example is Petri nets, a non-deterministic model that is well-suited for distributed systems with concurrent events. This model, extended with stochasticity, has been used together with Cellular Automata for event-based modeling of ecological systems (Gronewold and Sonnenschein, 1998). Other examples such as concurrent and process algebras provide tools for modeling parallel events with kinetics functions (normally adopted from the Gillespie algorithm) (Jordán et al., 2011).

Although each of these (extended) computational frameworks has been used as a modeling framework for many scenarios, none of them fully integrates the dynamics and structural details of complex systems (Colomer-Cugat et al., 2014). In this concern, P systems provide the following advantages as a computational modeling framework: (a) flexibility, i.e. minor changes in the system are reflected as minor changes in the model, (b) capability of simultaneous modeling of coexisting species and their interaction with the environment, (c) ability to define spatially-explicit dynamics (Colomer et al., 2013). For all of these, several membrane computing frameworks have been proposed, such as Dynamical Probabilistic $P$ (DPP) systems (Besozzi et al., 2008), Minimal Probabilistic P (MPP) systems (Barbuti et al., 2015) and Population Dynamics P (PDP) systems (Colomer et al., 2013; Colomer-Cugat et al., 2014). In what follows, we compare them with our proposed PGSP systems.

DPP systems were first introduced for modeling metapopulations (Besozzi et al., 2008). A DPP system consists of a rooted-tree structure with compartments. As in PGP systems, a DPP rule transforms a multiset $u$ in a compartment $i$ into a multiset $v$, which can be sent to another membrane $j$. Moreover, a kinetic constant is used to proportionally calculate the probability of application of the rule. The main differences with PGSP systems are: (a) the probabilities are computed per transition step, depending on the amount of objects present in the compartment, what is more computationally expensive for simulators, (b) it uses a rooted-tree structure instead of a directed-graph, and (c) compartments do not have contexts (flags in PGP systems).

MPP systems, introduced in 2015 (Barbuti et al., 2015), have been used to model the ecological problem of stability of European water frog populations. They are flat P systems (i.e. without an associated structure), whose rules have an associated multiset of promoters and a rate function. Rule promoters are used to represent contexts, and they can coexist in the same configuration. The rate function is used to calculate the probabilities for each transition step as a function of the number of individuals. Thus, the main differences with PGSP systems are: (a) no explicit representation of space, (b) probabilities are calculated in each transition step and used to select the sequence of rules to be applied, and (c) multiple promoters can coexist at a given instant. In order to allow 


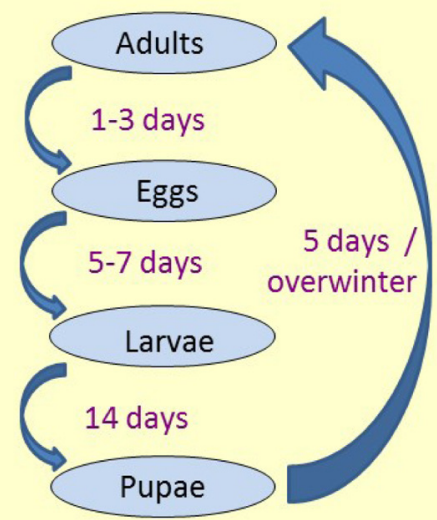

Fig. 2. Pieris oleracea life cycle.

the representation of spatial and other structural aspects, alternative variants have been proposed, such as Attributed Probabilistic $\mathrm{P}$ (APP) systems.

PDP systems are a class of multienvironment $\mathrm{P}$ systems with a probabilistic approach, where each environment contains a rootedtree structure of compartments (the same in all of them). In these systems, rules with the same left-hand side are applied following a multinomial distribution (Colomer-Cugat et al., 2014). This framework has been applied to model the dynamics of several animal species, such as of the zebra mussel Dreissena polymorpha (Colomer et al., 2014), the avian scavengers in the Pyrenees (Colomer et al., 2011b) and the Pyrenean Chamois (Rupicapra p. pyrenaica) (Colomer et al., 2011a). PGP systems (and so, PGSP) are multienvironment P systems (García-Quismondo et al., 2015) where each environment is elemental (i.e. do not contain a inner additional structure), and the contexts are represented by flags, and discriminate between evolution and context-changing rules. In turn, flags also provide an extended way to define a wider range of contexts per environment. Finally, PGSP systems enrich this framework with scripts, as described above.

\section{Methods}

\subsection{Ecological system}

We modeled a single-sex (female) population of the native $P$. oleracea, the green-veined white butterfly (Fig. 2) under various invasion scenarios. The species is generally bivoltine (two nonoverlapping generations during one breeding season), but can be trivoltine (Opler and Krizek, 1984; Opler et al., 1992; Klots, 1951; Scudder, 1889; Chew et al., 2012). The butterfly breeding season in our study area in the northeastern USA, near Lenox, Massachusetts (USA, $42^{\circ} 23^{\prime} \mathrm{N} ; 73^{\circ} 14^{\prime} \mathrm{W}$ ) starts with the first flight of adults in late April, and in recent years has extended to a partial fourth flight period (Herlihy et al., 2014). As is typical of butterflies, P. oleracea lays its eggs on several species of host plants to which its larvae are adapted, and then it dies; eggs subsequently grow through five instars (size classes) before pupating, to emerge later as adults of the next generation (Opler and Krizek, 1984; Opler et al., 1992; Klots, 1951; Scudder, 1889), and start a new breeding cycle the next spring.

The study was carried out on public lands and rights-of-way in Berkshire County near Lenox, Massachusetts under a permit to collect $P$. oleracea, a state-listed threatened species, from the Massachusetts Division of Fish and Wildlife 159.16SCI to Frances S.
Chew for 2016 (and its antecedents). Each year, as a condition of our permit, we file records with the Massachusetts Natural Heritage and Endangered Species Program of our activities and a detailed map showing where fieldwork was conducted.

In our study system, as the breeding season progresses, host plant species and abundances are dynamic in space and time (across butterfly generations within a breeding season). Specifically, we modeled 3 distinct habitat types: deep (beech-maple-hemlock) forest, the open field (a wet meadow) and an edge area transitioning between these two. The areas are identified because they have distinct plant phenologies that affect butterfly distributions and abundances within an annual cycle. We consider three nonoverlapping generations per year, in such a way that the butterfly generation in each one of the habitats is influenced by that habitat's seasonal ecological features. As an example, Cardamine diphylla Michx. is relatively abundant during the first generation in the forest, but drops to negligible levels during the second and third (Table 1). Earlier work suggests that parasitism by the exotic braconid wasp Cotesia glomerata L. is important in affecting butterfly population dynamics; $P$. oleracea was an unintended target of this parasitoid that was introduced in the late 19th century to control the important cabbage-worm ( $P$. rapae L.). However, beginning in the late 1980 s, a second exotic braconid wasp - a specialist on $P$. rapae - was introduced, and has apparently competitively excluded the first parasitoid (Herlihy et al., 2012), thus reducing predation pressure on the native butterfly (Herlihy et al., 2012; Morton et al., 2015).

In our model, the first two non-overlapping generations give rise to the next generation during the same breeding season. A fraction of butterflies successfully overwinter each generation, eventually emerging to become the adults starting the breeding season the following year (Fig. 2). We consider three different scenarios according to the characteristics of the two invasive plants $C$. pratensis $L$. (a perennial and good host for $P$. oleracea) and $A$. petiolata (a strict biennial and poor host, initially; $P$. oleracea larvae are now able to develop on leaf tissue of bolting plants, but not on the first-year rosettes). Differences in the abundances of these host species in each of the three habitats account for between-habitat dispersal patterns and population levels for $P$. oleracea. These phenomena are detailed in the Results section. In short, butterflies tend to cluster in sites with large numbers of good host plants. When good host plants are not available, butterfly numbers can dwindle if there is no alternative host plant or, if the only available plant is the poor host, A. petiolata combined with the selection regime that favors resistant genotypes able to develop successfully on it. Based on earlier work (see Chew et al., 2012; Keeler et al., 2006; Keeler and Chew, 2008 and references therein), it appears that the trait allowing $P$. oleracea larval development on $A$. petiolata is a single gene or gene complex that is inherited as a Mendelian dominant trait (Bowden, 1971). Consequently, for the model, we considered genotypes to be resistant ( $R R$ and $R r$ ) or the wild type, non-resistant ( $r r$ ); here, resistant means that individuals are able to thrive on this exotic plant. Table 1 describes the plant phenological occurrences and changes in abundance over time in all 3 habitats throughout the 3 different scenarios and the 3 modeled generations. $P$. oleracea larvae of genotype $r r$ develop well on all of these plant species except on $A$. petiolata.

\subsection{Probabilistic Guarded Scripted P(PGSP) systems}

We designed our population model using the Membrane Computing paradigm, that provides theoretical computational devices inspired by the structure and functioning of the living cell (Păun, 2000). The ability of cells to run multiple, distributed processes in parallel in a perfectly synchronized manner is a great source of computational inspiration to model real-life phenomena with 
Table 1

Qualitative representation of plant species abundances.

\begin{tabular}{|c|c|c|c|c|}
\hline Plant & Generation & Habitat & Scenario & Abundance \\
\hline Cardamine diphylla & 1 & Forest & Pre-invasion & Medium \\
\hline C. diphylla & 1 & Forest & Invasion & Low to medium \\
\hline C. diphylla & 1 & Forest & Post-invasion & Low \\
\hline C. pratensis & $1-3$ & Edge and field & Pre-invasion & Low \\
\hline C. pratensis & $1-3$ & Edge & Invasion & Low to high \\
\hline C. pratensis & $1-3$ & Field & Invasion & Low to high \\
\hline C. pratensis & $1-3$ & Edge & Post-invasion & High \\
\hline C. pratensis & $1-3$ & Field & Post-invasion & High \\
\hline Alliaria petiolata & 1 & Forest & Invasion & Low to medium \\
\hline Alliaria petiolata & 1 & Edge & Invasion & Low to high \\
\hline A. petiolata & 1 & Forest & Post-invasion & Medium \\
\hline A. petiolata & 1 & Edge & Post-invasion & High \\
\hline Brassica rapa \&crops & 2 & Field & All & Medium \\
\hline B. orthoceras \&Native mustards & 2 & Edge and field & Pre-invasion & Low to medium \\
\hline
\end{tabular}

the same characteristics. In this sense, processes modeled so far with membrane computing range from bio-molecular phenomena (Valencia-Cabrera et al., 2013; Romero-Campero and Pérez-Jiménez, 2008b,a) to swarm robotics (Vasile et al., 2012; García-Quismondo et al., 2013; García-Quismondo, 2014; Pavel and Buiu, 2012), fault diagnosis (Wang et al., 2015a,b; Peng et al., 2013), unsupervised learning (Peng et al., 2015b), image segmentation (Peng et al., 2014, 2015a) and population dynamics (Colomer et al., 2011b, 2013, 2014).

The seminal variant of membrane computing is structured after a cell on a hierarchy consisting of a rooted tree-like architecture of processor units, called membranes. The root membrane is known as the skin membrane. Each membrane delimits a region (the space between a membrane and the immediately inner membranes, if any) and contains objects (abstractions of chemical substances, individuals, food, etc.) expressed by a multiset over a common working alphabet. A multiset of objects is a set where the elements can appear repeated. In a region, objects can evolve or be sent through membranes according to given rewriting rules (associated with the regions), that are abstractions of chemical reactions, movements of individuals, etc. (see Păun, 2000 for more details).

Probabilistic Guarded P systems (PGP systems, for short) (García-Quismondo et al., 2015) provide a membrane computingbased modeling framework for population dynamics. Roughly speaking, a PGP system consists of a network of environments such that each environment is identified by a unique label and contains a multiset of objects of a common working alphabet. These objects represent the items (individuals, resources, etc.) involved in the system under study. New symbols can be produced by the application of rules associated with the environments. At any instant, each environment has an associated flag on its borderline that denotes its context (e.g. current season of the year in a model). Flags enable the model to switch the application of rules associated with each environment according to its context. The idea of a flag as a promoter symbol that enables the application of a rule is directly inspired by previous membrane computing models such as P systems with active membranes, in which a set of electrical charges (positive, negative, and neutral) switch the membrane between operation modes (Ipate et al., 2012; Song et al., 2014; Alhazov and PérezJiménez, 2007; Păun, 1999) or, more directly, kernel P systems, in which the existence or absence of objects in certain quantities determines the application of each rule. Moreover, the usage of functions capable of manipulating real values as a way to break through the intrinsic limitations of discrete rewriting rules has also been applied in other models in membrane computing (Pavel et al., 2010; Vasile et al., 2012; Hinze et al., 2007; Romero-Campero and Pérez-Jiménez, 2008b; Păun and Păun, 2006; García-Quismondo et al., 2013; Bernardini et al., 2006).

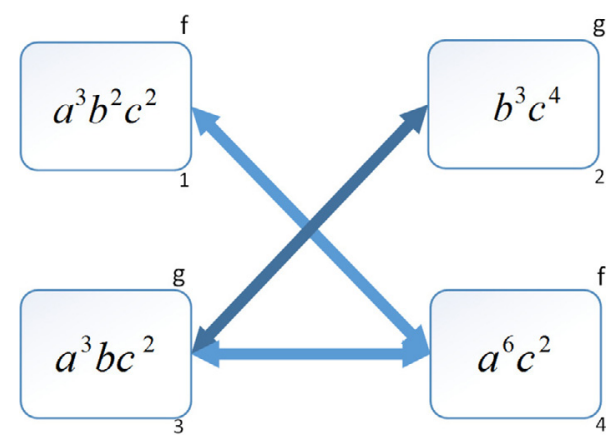

Fig. 3. A graphical representation of a general PGP system at a given instant.

A PGP system is depicted in Fig. 3. Each round-cornered rectangle represents an environment with an associated label (subscript) and flag (superscript). Environments are interconnected if and only if there exists at least one rule that sends objects between them. In these PGP systems, each environment contains a multiset of objects of different types, whose cardinality is denoted by a superscript. That is to say, multiset $a^{3} b^{2} c^{2}$ is a collection of objects containing three $a$ s, two $b s$ and two cs. Likewise, each environment has an associated label denoted as its subscript and a flag at each instant, which we represent as a superscript to the environment.

We can consider this PGP system as a model of a sample ecosystem. Such an ecosystem consists of 4 differentiated habitats, that we label with numbers 1 to 4 . In this example, we are interested in the population dynamics of 3 species, or 3 stages of individuals of the same species, and we represent these different classes as objects $a, b$ and $c$. Finally, we can also consider two different weather types (such as sunny and rainy) that heavily influence the dynamics of each environment, and represent them with flags $f$ and $g$. With all these ingredients in mind, we can say that environment 1 is interconnected solely with environment 4 and, at a given instant, is affected by weather $f$ and contains 3, 2 and 2 individuals of types $a, b$ and $c$, respectively.

Fig. 4 represents the model proposed in this work. This model considers 3 habitats (forest, edge and field), each one modeled as 3 environments: 2 for plants (good hosts C. diphylla and C. pratensis and poor host $A$. petiolata) and one for distribution. Only objects representing butterflies are present in the diagram; but $t_{1}$ for genotype $R r$, but $t_{2}$ for $R R$ and $b u t_{3}$ for $r r$. For instance, in the represented configuration the compartment Good host, Forest contains 320 butterflies of genotype $R r, 63$ of $R R$ and 782 of $r r$, where the compartment Bad host, Forest does not contain any butterfly. Each of the 3 modeled generations is divided in 4 stages, that is, $1 \leq i \leq 3 \wedge 1 \leq j \leq 4$. At every instant, all plants have the same associated gen $n_{i, j}$ flag, where $i$ and $j$ are respectively the generation and the stage. Likewise, all distri- 


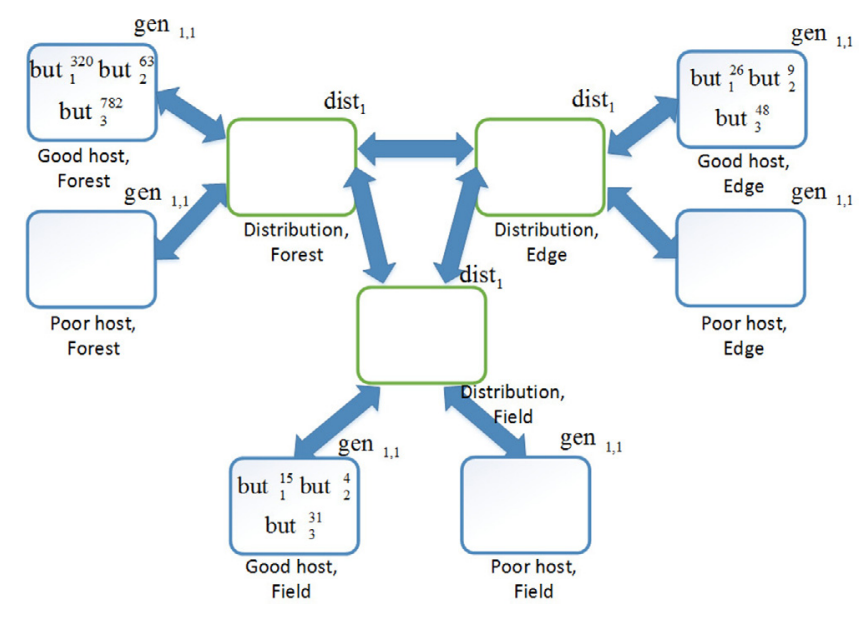

Fig. 4. Model configuration at early stages of the first generation. For optimized usage of available space, here environment labels are represented below each environment instead of as subscripts.

bution compartments have the same associated dist $t_{i}$ flag, where $i$ is the current generation.

In this paper, a variant of PGP systems known as Probabilistic Guarded Scripted P systems (PGSP systems, for short) is introduced as a framework to model and explore the conditions under which an allele conferring ability of $P$. oleracea larvae to develop on $A$. petiolata might have proliferated. PGSP systems consider an additional alphabet of computable functions (scripts) whose domain and range are the set of all multisets over the working alphabet. There exists a special script $\sigma$ that denotes the identity function, i.e. a function such that, given a multiset, it returns the same multiset. Scripts are a novel and exclusive ingredient of PGSP systems that serve as a shortcut for operations that are not intuitive by solely applying rewriting rules, such as processes whose probability of occurrence is a function on the number of individuals in an environment at a given instant. Examples of these are processes involving a habitat-specific carrying capacity.

Rules are mathematical constructs that abstract interactions taking place in the ecosystem under study. There exist two types of rules in the system: (a) rewriting rules; and (b) context-changing rules. Each rule consists of a left-hand side, representing the requirements of a rule to be applied, and a right-hand side, that describes the effect of the application of such a rule.

A rewriting rule $r$ is represented according to the following syntax: $r \equiv\{f\}(u)_{e_{j}} \stackrel{p(r)}{\longrightarrow}(v)_{e_{j_{1}}}$, where $f$ is a flag, $u$ and $v$ are multisets of objects, $e_{j}$ and $e_{j_{1}}$ are environments and $p(r)$ indicates the probability of application of the rule $r$ provided that it is applicable at a given instant. If $p(r)$ is equal to 1 , then we omit it. The summing of probabilities $p(r)$ of all rewriting rules sharing the same left-hand side must be equal to 1 . For each flag $f$ and environment $e_{j}$ there cannot exist two rewriting rules with overlapping but different lefthand sides. If environments $e_{j}$ and $e_{j_{1}}$ are the same, then we can also use the following notation: $r \equiv\{f\}(u \stackrel{p(r)}{\longrightarrow} v)_{e_{j}}$.

If in an area delimited by environment $e_{j}$, with flag $f$, we find a multiset of objects $u$, then the rule $r$ can be applied with a probability $p(r)$. When this rule is applied, the multiset of objects $u$ is removed from $e_{j}$ (the flag $f$ is not consumed) and the multiset of objects $v$ is produced into $e_{j_{1}}$. This approach of sending objects across environments and allowing them to change their class in the process is akin to that used in kernel P systems (Gheorghe et al., 2013a,b; Dragomir et al., 2014; Gheorghe and Ipate, 2014), PDP systems (Colomer et al., 2013) and, in a slightly different manner, in tissue-like P systems (Díaz-Pernil et al., 2007; Freund et al., 2005; Macías-Ramos et al., 2013).
A context-changing rule is described as $r \equiv\{f\}(u, f \longrightarrow v, g, s)_{e_{j}}$ where $f$ and $g$ are flags, $u$ and $v$ are multisets of objects, $e_{j}$ is an environment and $s$ is a script. If $s$ is the identity function, then we omit it. There can exist (at most) only one context-changing rule with the same left-hand side, and the probability of applying a context-changing rule given the necessary conditions is always 1 .

If in an area delimited by environment $e_{j}$, with flag $f$, we find a multiset of objects $u$, then the context-changing rule $r$ will be applied (even in the case where some rewriting rules are applicable). When applying this rule, objects in the multiset $u$ change their class into $v$ and the flag $f$ associated with $e_{j}$ is transformed into $g$, that is, flag $f$ is consumed, so that rule will be applied once at an instant.

If at the same time a context-changing rule and several rewriting rules are applicable to an environment $e_{j}$, then the contextchanging rule must be applied (only once) and rewriting rules will be applied to the remaining objects in a maximal way, that is, these objects are assigned to rules in such a manner that after the assignation no further rewriting rule can be applied. We suppose that in one transition step, context-changing and rewriting rules are applied first, and then the new multiset of objects associated with $e_{j}$ is obtained by applying the script to the multiset resulting from the application of the rules. After all rules have been applied, the multiset associated with each environment is updated according to its script at that instant, generating the next configuration in the computation.

A computation of a PGSP system is a (finite or infinite) sequence of configurations (instantaneous descriptions of the system) such that: (a) the first term is the initial configuration of the system; (b) for each $k \geq 2$, the $k$ th term of the sequence is obtained from the previous one in a transition step (Păun, 2000).

It is worth pointing out that the framework of PGSP systems is suited to model the dynamics of an ecosystem, a region in the space that encloses a number of distinct areas with certain characteristics (e.g. landscape, weather conditions, etc.). Within these areas, individuals develop conditioned by their own biological and demographic singularities. Individuals develop simultaneously, interacting and competing with each other for resources or mating and constrained by the limits imposed by their environment. This behavior can be abstracted as patterns or rewriting rules. Individuals can also move looking for more adequate habitats and be hindered by the physical restrictions inherent to movement (carrying capacity, natural barriers, distance, etc.). Each of these areas can be mapped to a different spatial environment in a PGSP model and its contents can be specified as an environment with its own geographical, biological and climatological particularities. In that situation, rewriting rules are abstractions of the movement and lifecycle dynamics of individuals among habitats represented by environments, whilst context-changing rules dictate how the specificities of each habitat, in conjunction with the biological rhythms of the animals, determine the fate of its inhabitants. In real ecosystems, processes are carried out simultaneously, synchronized and inter-related, dictated by overarching biological cycles of the organisms that compose them. We represent this synchronization with a global clock that marks the time of the whole system and a flag associated with each environment that represents the stages in these cycles.

\section{Computational modeling frameworks in population dynamics}

Traditionally, the study of complex systems (and population dynamics, in particular) has been addressed by using ordinary differential equations (ODEs). This approach shows in turn some drawbacks when used for discrete and stochastic processes, and poses some inflexibility to changes, that can cause a big impact 
in the defined equations (Costantino et al., 1997; Grimm, 1999; Grimm et al., 1999; Judson, 1994; Romero-Campero and PérezJiménez, 2008a). Hence, other computational paradigms have been considered to define more accurate models. One example is Petri nets, a non-deterministic model that is well-suited for distributed systems with concurrent events. This model, extended with stochasticity, has been used together with Cellular Automata for event-based modeling of ecological systems (Gronewold and Sonnenschein, 1998). Other examples such as concurrent and process algebras provide tools for modeling parallel events with kinetics functions (normally adopted from the Gillespie algorithm) (Jordán et al., 2011).

Although each of these (extended) computational frameworks has been used as a modeling framework for many scenarios, none of them fully integrates the dynamics and structural details of complex systems (Colomer-Cugat et al., 2014). In this concern, P systems provide the following advantages as a computational modeling framework: (a) flexibility, i.e. minor changes in the system are reflected as minor changes in the model, (b) capability of simultaneous modeling of coexisting species and their interaction with the environment, (c) ability to define spatially-explicit dynamics (Colomer et al., 2013). For all of these, several membrane computing frameworks have been proposed, such as Dynamical Probabilistic P (DPP) systems (Besozzi et al., 2008), Minimal Probabilistic P (MPP) systems (Barbuti et al., 2015) and Population Dynamics P (PDP) systems (Colomer et al., 2013; Colomer-Cugat et al., 2014). In what follows, we compare them with our proposed PGSP systems.

DPP systems were first introduced for modeling metapopulations (Besozzi et al., 2008). A DPP system consists of a rooted-tree structure with compartments. As in PGP systems, a DPP rule transforms a multiset $u$ in a compartment $i$ into a multiset $v$, which can be sent to another membrane $j$. Moreover, a kinetic constant is used to proportionally calculate the probability of application of the rule. The main differences with PGSP systems are: (a) the probabilities are computed per transition step, depending on the amount of objects present in the compartment, what is more computationally expensive for simulators, (b) it uses a rooted-tree structure instead of a directed-graph, and (c) compartments do not have contexts (flags in PGP systems).

MPP systems, introduced in 2015 (Barbuti et al., 2015), have been used to model the ecological problem of stability of European water frog populations. They are flat P systems (i.e. without an associated structure), whose rules have an associated multiset of promoters and a rate function. Rule promoters are used to represent contexts, and they can coexist in the same configuration. The rate function is used to calculate the probabilities for each transition step as a function of the number of individuals. Thus, the main differences with PGSP systems are: (a) no explicit representation of space, (b) probabilities are calculated in each transition step and used to select the sequence of rules to be applied, and (c) multiple promoters can coexist at a given instant. In order to allow the representation of spatial and other structural aspects, alternative variants have been proposed, such as Attributed Probabilistic $\mathrm{P}$ (APP) systems.

PDP systems are a class of multienvironment P systems with a probabilistic approach, where each environment contains a rootedtree structure of compartments (the same in all of them). In these systems, rules with the same left-hand side are applied following a multinomial distribution (Colomer-Cugat et al., 2014). This framework has been applied to model the dynamics of several animal species, such as of the zebra mussel D. polymorpha (Colomer et al., 2014), the avian scavengers in the Pyrenees (Colomer et al., 2011b) and the Pyrenean Chamois (R. p. pyrenaica) (Colomer et al., 2011a). PGP systems (and so, PGSP) are multienvironment P systems (García-Quismondo et al., 2015) where each environment is elemental (i.e. do not contain a inner additional structure), and
Table 2

Non-indexed parameters for Pieris napi oleracea model.

\begin{tabular}{|c|c|c|c|}
\hline Parameter & Description & Associated index & Value \\
\hline \multicolumn{4}{|c|}{ Size parameters } \\
\hline$n g$ & Number of generations considered & $i$ & 3 \\
\hline$n p$ & Number of plants considered & $k$ & 2 \\
\hline nge & Number of genotypes considered & $g$ & 3 \\
\hline nenv & Number of environments considered & pa & 3 \\
\hline ny & Number of years simulated & $y$ & 25 \\
\hline ns & Number of stages & $j$ & 4 \\
\hline$n m$ & Number of cycles for Stage 1 & l & 2 \\
\hline nls & Number of cycles for Stage 2 & $h$ & 10 \\
\hline$n c$ & Number of cycles for Stages 3 and 4 & $c$ & 3 \\
\hline про & Number of plant orders & $s$ & 4 \\
\hline Parameter & Description & Value & \\
\hline \multicolumn{4}{|c|}{ Other non-indexed parameters } \\
\hline$R$ & $\begin{array}{l}\text { Proportion homozygous butterflies } \\
\text { becoming heterozygous }\end{array}$ & 0.01 & \\
\hline$F$ & Proportion of migrating butterflies & 0.1 & \\
\hline$D$ & Proportion of ovipositing butterflies & 0.8 & \\
\hline$U$ & $\begin{array}{l}\text { Probability for larvae of becoming } \\
\text { pupae upon reaching the last instar }\end{array}$ & 0.83 & \\
\hline Sw & $\begin{array}{l}\text { Proportion of pupae surviving } \\
\text { overwinter }\end{array}$ & 0.169 & \\
\hline Ef & Number of eggs per female & 114 & \\
\hline
\end{tabular}

the contexts are represented by flags, and discriminate between evolution and context-changing rules. In turn, flags also provide an extended way to define a wider range of contexts per environment. Finally, PGSP systems enrich this framework with scripts, as described above.

\subsection{Model description}

We propose a population dynamics model based on Membrane Computing of the effect of the concurrent invasions of plants A. petiolata and $C$. pratensis on the genotype distribution and population numbers of the butterfly species $P$. oleracea. The modeled system consists of three different environments (parameter nenv represents the number of environments): forest, edge and open field. In each habitat, two types of plants (parameter $n p$ represents the number of types of plants) are considered: good hosts ( $C$. diphylla and $C$. pratensis) and bad host (A. petiolata). We consider a node per type of plant per habitat, plus an additional node per habitat for butterfly distribution. Therefore, if the total number of habitats is $n e n v=3$ and the total number of plant species is $n p=2$, the number of nodes in $G$ is nenv $\times(n p+1)$; in our case, $3 \times(2+1)=9$. We reserve the index $p a$ for environments and the index $k$ for plants. We consider 3 butterfly genotypes (parameter nge represents the number of genotypes): heterozygous, homozygous dominant and homozygous recessive. These genotypes are indexed by 1, 2 and 3 , respectively. In our model specification, we reserve the index $g$ for genotypes. We also consider 3 generations per year, and index them by $i$. We model each generation in 4 stages (parameter $n s$ represents the number of stages). The first stage consists of 2 transition steps (parameter $\mathrm{nm}$ represents the number of cycles for stage 1), the second stage consists of 10 transition steps (parameter $n l s$ represents the number of cycles for stage 2 ), and the third and fourth stages consist of 3 steps each (parameter $n c$ represents the number of cycles for stages 3 and 4). Therefore, each generation (parameter $n g$ represents the number of genrerations considered) is modeled in 18 transition steps, plus additional steps for synchronization. This model is simulated for a number of years (parameter ny represents the number of years). Each year is equivalent to 3 generations plus additional synchronization steps, which accrues to 70 steps per year. Tables 2 and 3 display all parameters used in the general 
Table 3

Indexed parameters for Pieris napi oleracea model.

\begin{tabular}{|c|c|}
\hline Parameter & Description \\
\hline \multicolumn{2}{|c|}{ General parameters } \\
\hline$N_{g}$ & Initial butterflies of phenotype $g$ \\
\hline Prop $_{i, k, p a}$ & Proportion of butterflies in plant $k$ in generation $i$ and environment $p a$ \\
\hline $\operatorname{Diap}_{p a}$ & Proportion of butterflies entering diapause in environment $p a$ \\
\hline$p_{i}$ & Proportion of recessive homozygous butterflies in generation $i$ \\
\hline$H_{i}$ & Hatching success for generation $i$ \\
\hline Hat $_{k, g}$ & Hatching success in plant $k$ and genotype $g$ \\
\hline Det $_{k, g}$ & Larvae adaptation of genotype $g$ to plant $k$, instar in and generation $i$ \\
\hline$O_{i}$ & Proportion of pupae entering diapause in generation $i$ \\
\hline$M_{i}$ & Proportion of pupae which emerge successfully in generation $i$ \\
\hline$M i_{p a_{1}, p a_{2}}$ & Proportion of migrating butterflies from patch $p a_{1}$ to patch $p a_{2}$ \\
\hline Cost $_{i, g}$ & Detoxification cost in generation $i$ and genotype $g$ \\
\hline$s i_{p a, i}$ & $\begin{array}{l}\text { Order of plants in patch pa and generation } i \text {. This is an tentative measurement of the plant } \\
\text { population; the greater the order, the larger the plant population in a patch. }\end{array}$ \\
\hline$K f_{s}, K l_{s}$ & Parameters denoting the amount of plants of order $s$ \\
\hline $\operatorname{Exp}_{y, p a}$ & Expansion of $A$. petiolata in relation with C. diphylla and C. pratensis in year $y$ and patch $p a$ \\
\hline$w e_{y, p a}$ & Overall growth of the host population (including good and bad hosts) in year $y$ and patch $p a$ \\
\hline
\end{tabular}

description of the model, their associated indexes and the specific values considered in our model.

The model consists of a PGSP system defined as follows:

$\Pi=\left(G, \Sigma, \Phi, \Theta, T,\left\{\left(f_{j}, E_{j}\right) \mid 1 \leq j \leq m\right\}, \mathcal{R}\right)$

where

- $G=(N, E)$ is a directed graph with a node per environment. The set of nodes is: $N=\{(k, p a) \mid 1 \leq k, p a \leq 3\}$, and the set of edges is: $E=\{\{(1, p a),(k+1, p a)\} \mid 1 \leq p a \leq 3 \wedge 1 \leq k \leq 2\} \cup\{\{(1, p a)$,

$\left.\left.\left(1, p a^{\prime}\right)\right\} \mid 1 \leq p a, p a^{\prime} \leq 3 \wedge p a \neq p a^{\prime}\right\}$

- The working alphabet $\Sigma$ is:

$$
\begin{aligned}
& \left\{\text { but }_{g}, \text { butl }_{g}, \text { butla }_{g}, \text { butd }_{g}, \text { emi }_{g}, \text { imm }_{g}, \text { egg }_{g}, \text { pupa }_{g}, \text { pupad }_{g}\right. \\
& \mid 1 \leq g \leq 3\} \cup\left\{\text { butla }_{i, g} \mid 1 \leq i, g \leq 3\right\} \cup\left\{c l_{j} \mid 1 \leq j \leq 10\right\} \cup\left\{c y_{k}\right. \\
& \mid 1 \leq k \leq n y\} \cup\{\text { dist }, \text { rst }\} \cup\left\{p l_{s} \mid 1 \leq s \leq 4\right\}
\end{aligned}
$$

and its objects represent the following entities (see Table 2 for details):

- Objects but , $_{\text {, butl }}$, butla butla $_{i, g}$, egg $g_{g}$ and pupa, $1 \leq g \leq 3$, $1 \leq i \leq 3$, represent butterflies, eggs and pupae of genotype $g$ respectively. Objects butl $_{g}$ and butla $_{i, g}$ represent females about to oviposit and those which have already oviposited. Objects butd $_{g}$ represent butterflies in diapause, and objects emi end $_{ } \mathrm{im}_{\mathrm{g}}$ represent emigrants and immigrants. Finally, objects pupad represent pupae which have entered diapause.

- Objects $\mathrm{Cl}_{j}, 1 \leq j \leq 10$, mark the time necessary for each development stage to be completed.

- Objects $c y_{k}, 1 \leq k \leq n y$, indicate the current year simulated.

- Object dist triggers the redistribution of butterflies among plants. This distribution pattern varies among generations.

- Object rst restarts the clock for Stage 2.

- Objects $p l_{s}, 1 \leq s \leq 4$ denote plant abundance of order $s$.

- Alphabet $\Phi=\left\{\right.$ gen $\left._{i, j}, \quad 1 \leq i \leq 4, \quad 1 \leq j \leq 5\right\} \cup\left\{\right.$ dist $_{i}, \quad$ iss $_{i}, \quad$ wait $_{i}$, $1 \leq i \leq n g\}$ is composed of flags that encode for the current stage of the model. These flags represent the following entities:

- Flags gen $_{i, j}, 1 \leq i \leq 4,1 \leq j \leq 5$, represent the current generation and stage in the model, eggs and pupae of genotype $g$, respectively.

- Flags dist $t_{i}, i_{i} s_{i}$ and $w_{a i t}, 1 \leq i \leq 3$, denote the time in which butterflies are redistributed from cell 1 to the rest of the cells. Similarly, objects $i s s_{i}, 1 \leq i \leq 3$, indicate the time for butterflies to migrate to cells $k, 1<k \leq 3$, for redistribution. Finally, objects wait $_{i}, 1 \leq i \leq 3$, indicate cells $k, 1<k \leq 3$, to issue a signal dist to cell 1 in preparation for butterfly redistribution.

- Alphabet $\Theta=\left\{s c_{s r}, 1 \leq s r \leq 2\right\} \bigcup\{\sigma\}$ is composed of scripts that represent computable functions which complement rule applications: for every multiset of $\Sigma u$, let $|u|_{a}, a \in \Sigma$ be the number of objects of type $a$ in $u$. Then, $s c_{1}(u)$ is defined as follows (Eqs. (1)-(6))

$\left|s c_{1}(u)\right|_{\operatorname{egg}}=2 \cdot p \cdot q \cdot \operatorname{sum} \cdot E f$

$\left|s c_{1}(u)\right|_{\text {egg }}=p^{2} \cdot \operatorname{sum} \cdot E f$

$\left|s c_{1}(u)\right|_{\operatorname{lgg}_{3}}=q^{2} \cdot \operatorname{sum} \cdot \mathrm{Ef}$

$\left|s c_{1}(u)\right|_{\text {butlag }}=|u|_{\text {butlg }} \cdot(1-F)$

$\left|s c_{1}(u)\right|_{\text {emig }}=|u|_{\text {butlg }} \cdot(F)$

$\left|s c_{1}(u)\right|_{\text {butlg }}=0$

for $1 \leq \mathrm{g} \leq 3$, where:

sum $=\sum_{g=1}^{3}|u|_{\text {butlg }}$

$p=\left(2 \cdot|u|_{\text {butl }_{2}}+|u|_{\text {butl }_{3}}\right) /(2 \cdot$ sum $)$

$q=1-p, \mu=\operatorname{sum} / K l_{s}$

$P(y)=\left(\mu^{y} \cdot e^{-\mu}\right) / y !$

$F=\left(P(0)-(P(0)-P K f)\left(\operatorname{sum} / K f_{s}\right)^{B}\right)$

for $0 \leq s \leq 2$. For any other object $a \in \Sigma$ such that $a$ is different from $\left\{\operatorname{egg}_{1}\right.$, egg $_{2}$, egg $_{3}$, butla $_{g}$, emi $_{g}$, butl $\left._{g}\right\},\left.s c_{1}(u)\right|_{a}=|u|_{a}$. Likewise, for each $u \in M(\Gamma), s c_{2}(u), u \in M(\Gamma)$ is computed identically to $s c_{1}(u)$, with the exception of the function $P(y)$, that is defined as: $P(y)=$ $(k+y-1) ! /(y ! \cdot(k-1) !) \cdot \begin{gathered}\mu \\ k+\mu\end{gathered} \cdot \begin{gathered}k^{y} \\ k+\mu\end{gathered} \quad$ in this case.

The aim of these equations is to mimic the aspects of the previous model (Keeler et al., 2006) that are too complex to be reproduced using rewriting rules. The overall number of plants in this scenario is not constant either, but increases with the passage of years. To represent this, values $K f_{s}$ and $K l_{s}$ are multiplied by a parameter we $w$,pa. Therefore, in this scenario the expressions of $\mu$ and $F$ appearing in function $s c_{1}(u)$ are replaced by $\mu=s u m /\left(K l_{s} \cdot w e_{y, p a}\right)$ and $F=$ $\left(P(0)-(P(0)-P K f)\left(\operatorname{sum} /\left(K f_{s} \cdot w e_{y, p a}\right)\right)^{B}\right)$. Likewise, in the inva- 
sion scenario the expression $\mu=K l_{s} /\left(\sum_{g=1}^{3}\left|u^{\prime}\right|_{\text {butlg }}\right)$ appearing in function $s c_{2}(u)$ is replaced by $\mu^{\prime}=\mu \cdot w e_{y, p a}$.

- The initial multisets are $\mathcal{M}_{1, p a}=\left\{b u t_{g}^{N_{g}}\right\}$ and $\mathcal{M}_{k, p a}=$ $\left\{c l_{1}, c y_{1}\right\}$, for $2 \leq k \leq 3,1 \leq p a \leq 3$. $\mathcal{M}_{1, p a}$ contains an initial number of butterflies that will be distributed across plants, and $\mathcal{M}_{k, p a}$ encodes for the initial year and clock cycle.

- The initial flags are $f_{1, p a}=$ dist $_{1}$ and $f_{k, p a}=$ gen $_{1,1}$, for $2 \leq k \leq 3$, $1 \leq p a \leq 3$.

- The finite set of rules in $\mathcal{R}$ is the following. First, the rules directly related with processes regarding $P$. oleracea life-cycle are described, followed by the synchronization rules. For the sake of simplicity, probabilities equal to 1 are omitted:

- First, butterflies are distributed among plants in each environment: $\left\{\right.$ dist $\left._{i}\right\}\left(\text { but }_{g}\right)_{1, p a} \stackrel{\text { Prop }_{i, k-1, p a}}{\longrightarrow}\left(\text { but }_{g}\right)_{k, p a}$, for $1 \leq i \leq 3,1 \leq$ $g \leq 3,2 \leq k \leq 3,1 \leq p a \leq 3$.

- In the invasion scenario, the abundance of plants on each patch increases over time. To represent this, in this specific scenario each appearance of any flag of the type dist $t_{i}, 1 \leq i \leq 3$ is replaced by other of the type dist $t_{i, y}, 1 \leq i \leq 3,1 \leq y \leq n y$, representing the growth rate. Consequently, each rule in the previous system whose flag is of type dist $t_{i}$ is transformed into ny rules (one per simulation year). Moreover, the flag associated with each environment $k, p a, 2 \leq k \leq 3,1 \leq p a \leq 3$ becomes dist $t_{1,1}$ instead of $d i s t_{1}$. In order to represent the replacement of native host $C$. diphylla by the invading species $A$. petiolata and $C$. pratensis, the rules: $\left\{\right.$ dist $\left._{i}\right\}\left(\text { but }_{g}\right)_{1, p a}\left(\text { but }_{\mathrm{g}}\right)_{k, p q}$ are replaced by the following sets of rules: $\left\{\right.$ dist $\left._{1, y}\right\}$ ( but $\left._{g}\right)_{1}$ prg $_{1, k-p_{1, k-1, p a}} \cdot$ Ext $_{y, p a}$

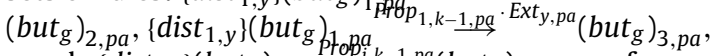

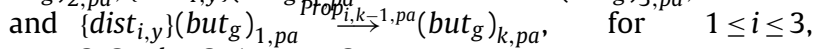
$1 \leq \mathrm{g} \leq 3,2 \leq k \leq 3,1 \leq p a \leq 3$.

- Then, the distribution flag is updated by the following rules: $\left\{\right.$ dist $\left._{i}\right\}\left(\text { dist }^{n p}, \text { dist }_{i} \longrightarrow \text { dist }_{i+1}\right)_{1, p a}$, for $1 \leq i \leq 3,1 \leq p a \leq 3$.

These rules synchronize the model, and do not reflect a biological process.

- Some homozygous butterflies mutate into heterozygous with probability $R: \quad$ gen $\left._{i, 1}\right\}\left(\text { but }_{g} \stackrel{R}{\longrightarrow} b u t_{1}\right)_{k, p a}$ and $\left\{\right.$ gen $\left._{i, 1}\right\}\left(\text { but }_{g} \stackrel{1-R}{\longrightarrow} \text { but }_{g}\right)_{k, p a}$, for $1 \leq i \leq 3, \quad 1 \leq g \leq 3, \quad 2 \leq k \leq 3$, $1 \leq p a \leq 3$.

- A proportion $\operatorname{Diap}_{p a}$ of butterflies enter diapause, and a pro-

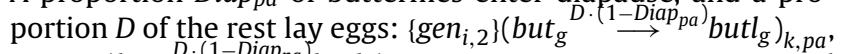

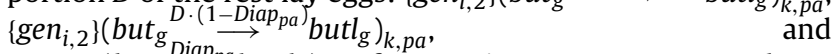
$\left\{\right.$ gen $\left._{i, 2}\right\}$ (butg $^{\text {Diap }_{p a}^{a}}$ butd $\left._{g}\right)_{k, p a}$, for $1 \leq i \leq 3, \quad 1 \leq g \leq 3, \quad 2 \leq k \leq 3$, $1 \leq p a \leq 3$.

- Eggs hatch into pupae with a probability that depends on their generation: fgen $\left._{1, j}\right\}\left(\operatorname{egg}_{g}{ }_{H_{2}} \cdot H_{1} \cdot \text { Hat }_{k-1, g} \cdot \text { Det }_{k-1, g} \cdot U \cdot\left(1-\text { Cost }_{i, g}\right)\right)_{k, p a}$, $\left\{\right.$ gen $\left._{i, j}\right\}\left(\operatorname{egg}_{g} \mathrm{H}_{2} \cdot \text { Hat }_{k-1, g} \cdot \text { Det }_{k-1, g} \cdot U \cdot\left(1-\text { Cost }_{i, g}\right)_{\text {pupa }}\right)_{k, p a}$, \left. and ${\left.\text { \{ } \text { gen }_{i, j}\right\}\left(\operatorname{egg}_{g} 1-H_{2} \cdot \text { Hat }_{k-1, g} \cdot \text { Det }_{k-1, g} \cdot U \cdot\left(1-\text { Cost }_{i, g}\right.\right.}_{\longrightarrow}\right)_{k, p a}$, for $1 \leq i \leq 3,1 \leq \mathrm{g} \leq 3,2 \leq k \leq 3,1 \leq p a \leq 3,3 \leq j \leq 4$.

- Pupae emerge as butterflies, die or enter diapause: \{gen $\left._{i, 5}\right\}\left(\text { pupa }_{g} \stackrel{\left(1-O_{i}\right) \cdot M_{i}}{\longrightarrow} \text { but }_{g}\right)_{k, p a}$, gen $\left._{i, 5}\right\}\left(\right.$ pupa $_{g}\left(1-\dot{O}_{i}\right) \cdot\left(1-M_{i}\right)_{k, p a}$, and $\left\{\right.$ gen $\left._{i, 5}\right\}\left(\text { pupa }_{g} \stackrel{O_{i}}{\longrightarrow} \text { pupad }_{g}\right)_{k, p a}$, for $1 \leq i \leq 3, \quad 1 \leq g \leq 3$, $2 \leq k \leq 3,1 \leq p a \leq 3$.

- Some butterflies disperse across environments or leave the system. Their proportion depends on the origin and destination of the migrants:

$\left\{\right.$ gen $\left._{i, 3}\right\}\left(\text { emi }_{g}\right)_{k_{1}, p a_{1}}{M i p a_{1}, p a_{2}}^{\longrightarrow}$ Prop $_{i, k_{2}-1, p a_{2}}\left(\mathrm{imm}_{\mathrm{g}}\right)_{k_{2}, p a_{2}}$, gen $\left._{i, 4}\right\}$ $\left(\text { emi }_{\mathrm{g}} \longrightarrow\right)_{k_{1}, p a_{1}}$, and $\left\{\right.$ gen $\left._{i, 4}\right\}\left(\mathrm{imm}_{\mathrm{g}} \longrightarrow\right)_{k_{1}, p a_{1}}$, for $1 \leq i \leq 3$, $1 \leq g \leq 3,2 \leq k_{1}, k_{2} \leq 3,1 \leq p a_{1}, p a_{2} \leq 3, p a_{1} \neq p a_{2}$.
- Butterflies laying eggs become butterflies which have already laid them. Notice that butterflies only lay eggs one single generation (their lifespan is around two weeks), and in the model they lay all the eggs on a single time for the sake of simplification. Moreover, remaining eggs are removed from the system:

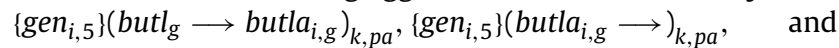
$\left\{\operatorname{gen}_{i, 5}\right\}\left(\operatorname{egg}_{g} \longrightarrow\right)_{k, p a}$, for $1 \leq i \leq 3,1 \leq g \leq 3,2 \leq k \leq 3,1 \leq p a \leq 3$. - Plant sizes are flushed and issue objects for distributing butterflies are generated: $\left\{g n_{i, 5}\right\}\left(p l_{s i_{p a, i}} \longrightarrow\right)_{k, p a}$, and $\left\{\right.$ gen $\left._{i, 6}\right\}\left(\text { gen }_{i, 6} \longrightarrow i s s_{i}\right)_{k, p a}$, for $1 \leq i \leq 3,2 \leq k \leq 3,1 \leq p a \leq 3$.

- Only diapausing pupae can survive overwinter. Therefore, butterflies, pupae (not overwintering) and eggs die: $\left\{\right.$ gen $\left._{n g+1,1}\right\}\left(\text { but }_{g} \longrightarrow\right)_{k, p a},\left\{\right.$ gen $\left._{n g+1,1}\right\}\left(\text { egg }_{g} \longrightarrow\right)_{k, p a},\left\{\right.$ gen $\left._{n g+1,1}\right\}$ $\left(\text { pupa }_{g} \longrightarrow\right)_{k, p a},\left\{\right.$ gen $\left._{n+1,1}\right\}\left(\text { but }_{g} \longrightarrow\right)_{k, p a}$,

and $\left\{\right.$ gen $\left._{n g+1,1}\right\}\left(\text { butl }_{g} \longrightarrow\right)_{k, p a}$, for $1 \leq g \leq 3, \quad 2 \leq k \leq 3$, $1 \leq p a \leq 3$.

- Diapausing pupae and butterflies that survive overwinter transform into non-diapausing butterflies with probability $S w$ : $\left\{\right.$ gen $\left._{n+1,1}\right\}\left(\text { pupad }_{g} \stackrel{S w}{\longrightarrow} \text { but }_{g}\right)_{k, p a},\left\{\right.$ gen $\left._{n g+1,1}\right\}\left(\text { pupad }_{g} \stackrel{1-S w}{\longrightarrow}\right)_{k, p a}$, $\left\{\right.$ gen $\left._{n g+1,1}\right\}\left(\text { butd }_{g} \stackrel{\text { Sw }}{\longrightarrow} \text { but }_{g}\right)_{k, p a}$,

and $\left\{\right.$ gen $\left._{n g+1,1}\right\}\left(\text { butd }_{g} \stackrel{1-S w}{\longrightarrow}\right)_{k, p a}$, for $1 \leq i \leq 3,1 \leq g \leq 3,2 \leq$ $k \leq 3,1 \leq p a \leq 3$.

- On each transition between generations, butterflies are redistributed: $\left\{\right.$ iss $\left._{i}\right\}\left(\text { but }_{g}\right)_{k, p a} \longrightarrow\left(\text { but }_{g}\right)_{1, p a}$, for $1 \leq i \leq 3,1 \leq g \leq$ $3,2 \leq k \leq 3,1 \leq p a \leq 3$.

- Additionally, some rules synchronize the model. These rules synchronize butterfly redistribution and update the current clock, generation and year (Eqs. (12)-(29)):

$\left\{\right.$ iss $\left._{i}\right\}\left(\text { iss }_{i} \longrightarrow \text { dist, } \text { wait }_{i}\right)_{k, p a}$

$\left\{\right.$ wait $\left._{i}\right\}(\text { dist })_{k, p a} \longrightarrow(\text { dist })_{1, p a}$,

for $1 \leq i \leq 3,2 \leq k \leq 3,1 \leq p a \leq 3$

$\left\{\right.$ wait $\left._{i}\right\} \quad\left(c_{c} \longrightarrow c l_{c+1}\right)_{k, p a}$,

for $1 \leq i \leq 3,2 \leq k \leq 3,1 \leq p a \leq 3,1 \leq c<3$

$\left\{\right.$ wait $\left._{i}\right\}\left(\mathrm{cl}_{3}, \text { wait }_{i} \longrightarrow \mathrm{cl}_{1}, \text { gen }_{i+1,1}\right)_{k, p a}$,

for $1 \leq i \leq 3,2 \leq k \leq 3,1 \leq p a \leq 3$

$\left\{\operatorname{gen}_{i, j}\right\}\left(c l_{c} \longrightarrow c l_{c+1}\right)_{k, p a}$,

for $1 \leq i \leq 3,2 \leq k \leq 3,1 \leq p a \leq 3,1 \leq c<3,3 \leq j \leq 4$

$\left\{\mathrm{gen}_{i, 1}\right\} \quad\left(\mathrm{cl}_{l} \longrightarrow \mathrm{cl}_{l+1}\right)_{k, p a}$,

for $1 \leq i \leq 3,2 \leq k \leq 3,1 \leq p a \leq 3,1 \leq l<2$

$\left\{\right.$ gen $\left._{i, 2}\right\} \quad\left(c l_{h} \longrightarrow c l_{h+1}\right)_{k, p a}$,

for $\leq i \leq 3,2 \leq k \leq 3,1 \leq p a \leq 3,1 \leq h<10$

$\left\{\operatorname{gen}_{i, j}\right\}\left(\mathrm{cl}_{3}, \operatorname{gen}_{i, j} \longrightarrow c l_{1}, \operatorname{gen}_{i, j+1}\right)_{k, p a}$,

for $1 \leq i \leq 3,2 \leq k \leq 3,1 \leq p a \leq 3,3 \leq j \leq 4$

$\left\{\right.$ wait $\left._{i}\right\}\left(c l_{n c}, \text { wait }_{i} \longrightarrow c l_{1}, \text { gen }_{i+1,1}\right)_{k, p a}$

$\left\{\right.$ gen $\left._{i, 1}\right\} \quad\left(c l_{n m}, \operatorname{gen}_{i, 1} \longrightarrow \operatorname{gen}_{i, 2}, p l_{s i_{p a, i}}\right)_{k, p a}$ 


\section{Generation 1}
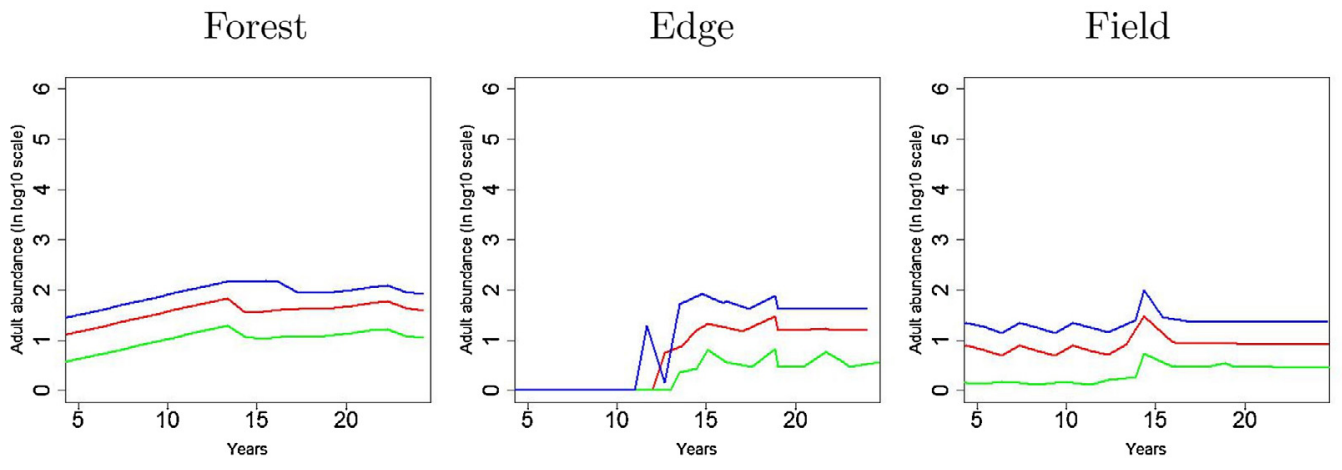

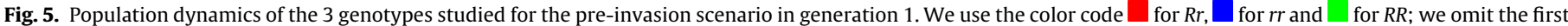
5 years in each plot to allow the dynamics of the system to stabilize.

$\left\{\right.$ gen $\left._{i, 2}\right\} \quad\left(c_{10}, \text { gen }_{i, 2} \longrightarrow s c_{\min (i, 2)}, \text { gen }_{i, 3}\right)_{k, p a}$

for $1 \leq i \leq 3,2 \leq k \leq 3,1 \leq p a \leq 3$.

We have simulated this model using P-Lingua (GarcíaQuismondo et al., 2010), a software tool for membrane computing. P-Lingua is a Java (Oracle Corporation, 2017) API that provides a plugin-based architecture to integrate new membrane computing frameworks in a straightforward manner, as well as a description language for defining $\mathrm{P}$ systems on plain text files. We specified the model according the P-Lingua syntax for PGP systems (GarcíaQuismondo et al., 2015). We have developed a simulator for PGSP systems and integrated them into P-Lingua. We have also developed a new graphical user interface (GUI) for P-Lingua known as MeCoGUI (García-Quismondo, 2014). This tool is available at the PLingua website (Garcia-Quismondo, 2017). MeCoGUI takes as input a P system specification in P-Lingua and a comma-separated values (CSV) file with its parameter values and outputs the same P system with its parameters instantiated in any of the supported output formats. It also simulates the P system and outputs the resulting computations. Finally, we also developed a $\mathrm{C}++$ simulator for PGSP systems that communicates with P-Lingua, and applied it to run our simulations. This simulator is available at the PMC GPU website (Martinez, 2013).

We ran simulations for different scenarios, that include: preinvasion (A. petiolata is absent and $C$. diphylla is present in medium levels of abundance), invasion (A. petiolata invades forest and edge habitats, $C$. diphylla declines to low abundance in all habitats and $C$. pratensis grows in number in the edge and the field) and postinvasion ( $A$. petiolata and $C$. pratensis stabilize in large numbers while $C$. diphylla remains in low levels of abundance). We set to $10 \%$ the percentage of butterflies that leave their habitat and disperse into another one, but also simulated scenarios with values for this parameter between $0 \%$ and $20 \%$. Each scenario was simulated 1000 times for a timespan of 25 years each, and average butterfly populations for each genotype have been calculated. Likewise, during the first 5 years of each scenario the system displays an unstable behavior, so we set the burn-in time to 5 years and omitted them to clarify our graphical observations. The model parameters and their values are available at the end of this work as an appendix.

\section{Results}

In this section, we present simulation results from three different scenarios: pre-invasion, invasion and post-invasion. We present plots describing the evolution of the genotypic profile in each environment in a representative sample of generations. We consider the first 5 years in each scenario as burn-in iterations that are necessary to stabilize the system's dynamics, and omit them from each plot.

\subsection{Pre-invasion}

In the pre-invasion scenario (Fig. 5), the populations of butterflies in all environments stay at basal levels. In this scenario, a stable number of $C$. diphylla is present but there is no A. petiolata in the system. As expected, a high frequency of the homozygous wildtype susceptible genotype ( $r r)$ occurs; because in the absence of $A$. petiolata there is no bias in favor of any allele, and, at the beginning of the simulated scenario, the $r r$ genotype is substantially the most numerous; at a proportion of approximately $15 \mathrm{rr}$ individuals versus $1 R R$ and $1 R r$ butterflies, based on the model by Morton et al. (2015). The forest habitat allows survival and proliferation of $R R, R r$ and $r r$ butterflies during the first generation of every year. However, the only plants left in the forest in generations 2 and 3 are $A$. petiolata rosettes, which are completely lethal to the butterfly larvae. This makes the forest unable to host butterflies, that are forced out of this habitat. In generations 2 and 3, individuals can find suitable hosts only in the edge and field habitats (Chew, 1981).

\subsection{Invasion}

In the invasion scenario, a growth of the populations in all environments is fueled by the numerical increase of $A$. petiolata and C. pratensis over time and their availability as host plants (Fig. 6). In the following figures, the upper row represents butterfly abundance values in $\log _{10}$ scale, while the lower row represents the evolution over time of the distribution of plants. For each invasion scenario, we only represent the first generation because plant abundances remain the same during the second and third generations. The exception is A. petiolata, that is present only in the first generation. As only $R R$ and $R r$ larvae can reach adulthood on $A$. petiolata bolting stalks, the proportions of these genotypes increase steadily at the expense of $r r$ genotypes. In this scenario, we incorporate a time delay of 10 years between the successive invasions of A. petiolata first and C. pratensis later in the edge habitat. An interesting phenomenon is that, as the growing number of plants in the field environment permits to host a greater number of butterflies during generations 2 and 3 each year, a significant number of individuals move from the forest to the field. Since the majority of host plants in the forest during generation 1 are $A$. petiolata, the allele $R$ increases its proportion. However, A. petiolata is absent in the open field. Therefore, there is no local bias for the allele $r$, the mixture of alleles $R$ and $r$ becomes significant, and the genotype $R r$ emerges.

We also tested the hypothesis that $C$. pratensis provided a way for the $P$. oleracea population to expand into the field habitat. To do 


\section{Generation 1}

Forest
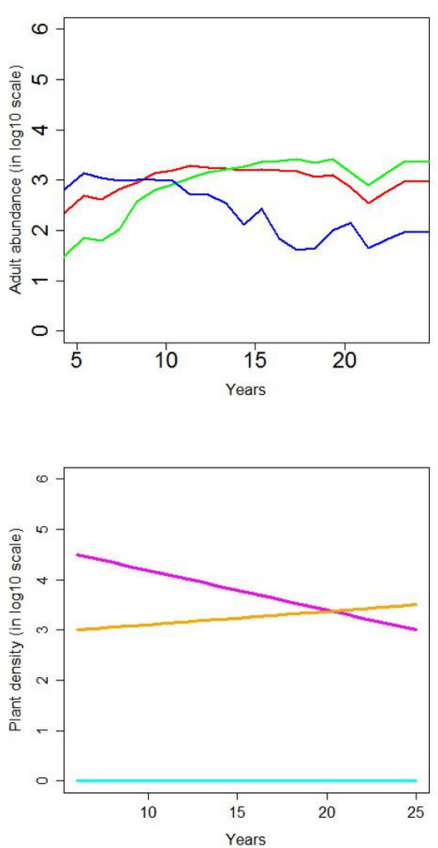

Edge
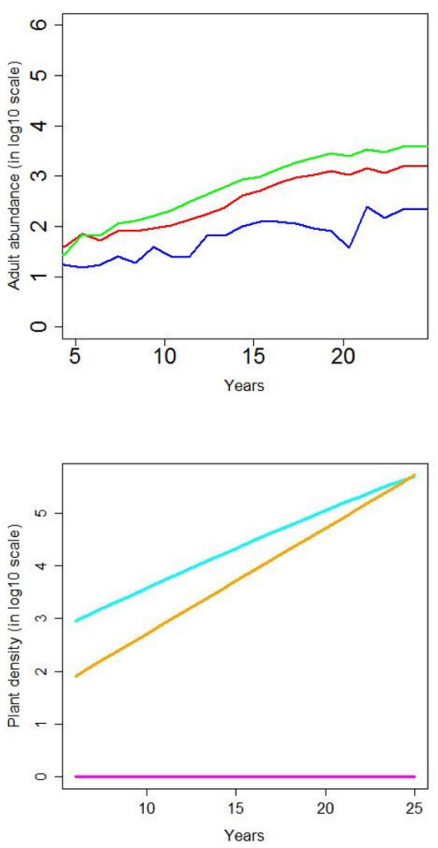

Field
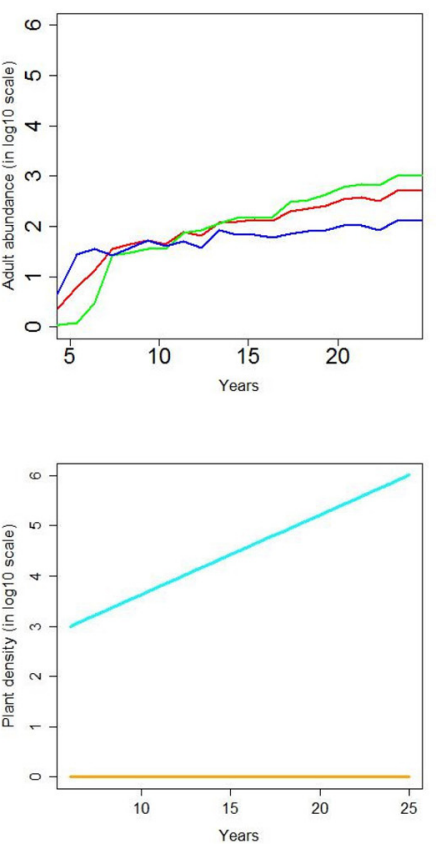

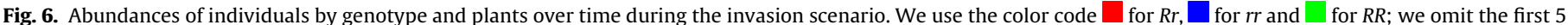
years in each plot to allow the dynamics of the system to stabilize. Likewise, we use the color code $\square$ for C. diphylla, $\square$ for C. pratensis and $\square$ for A. petiolata.

\section{Forest (Generation 1)}
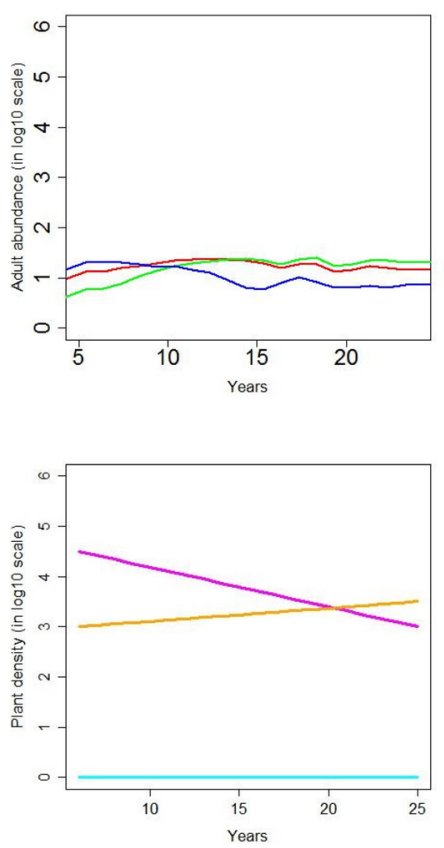

Edge (Generation 1)
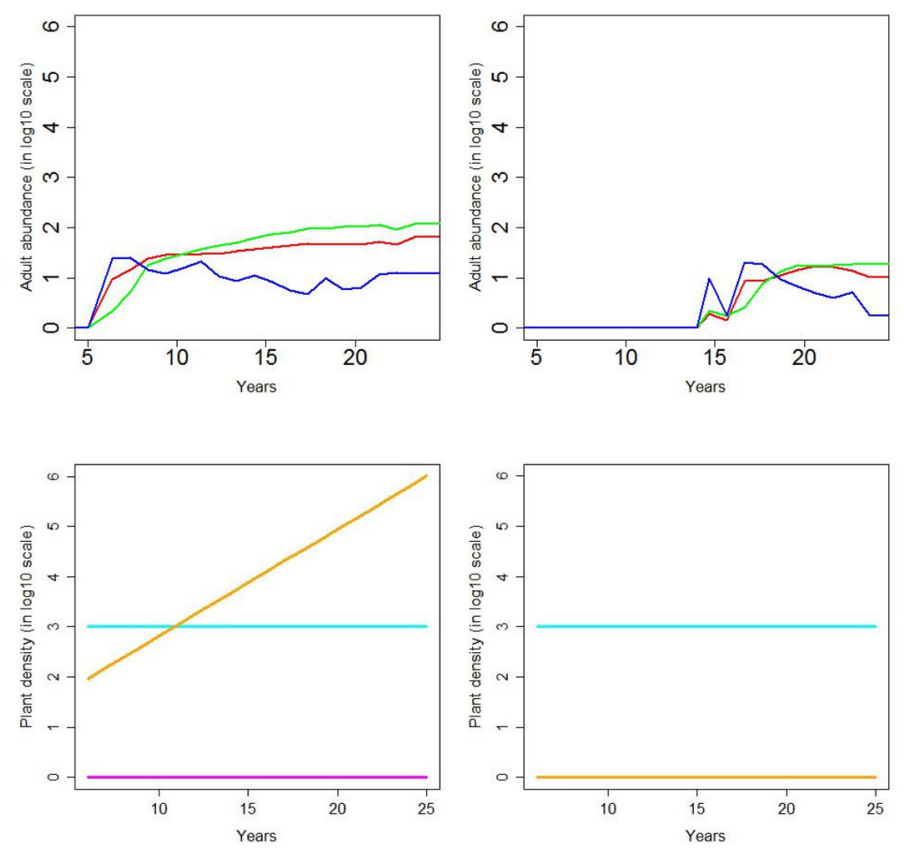

Field (Generation 2)

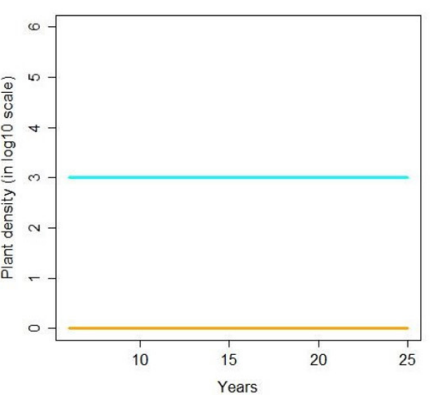

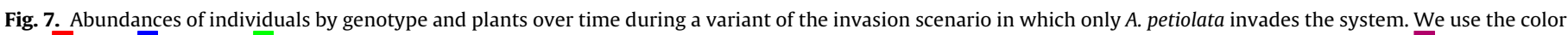

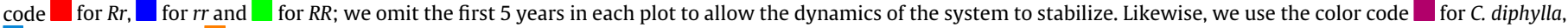
for C. pratensis and for A. petiolata.

so, we have simulated a scenario in which only $A$. petiolata invades the system, with no presence of $C$. pratensis (Fig. 7). As a result, the populations of $R R$ and $R r$ genotypes grow to similar levels to the $r r$ genotype, but the number of the latter does not decrease with respect to the initial scenario because the number of $C$. diphylla does not decrease. As an interesting effect, the field receives a significant number of $A$. petiolata-resistant individuals from the forest and the edge. Since the absolute number of plants is larger in the field and the edge during generations 2 and 3, butterflies do not disperse from the edge into the field until a critical mass is reached. However, unlike the previous scenario, the population of butterflies stabilizes 


\section{Generation 1}

Forest
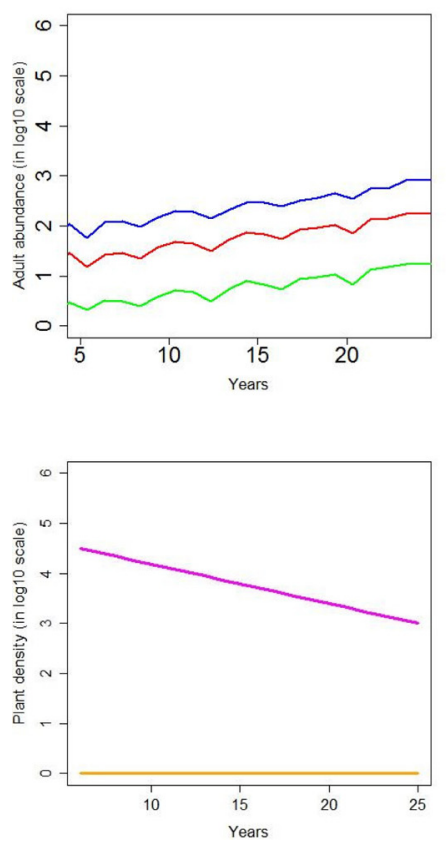

Edge
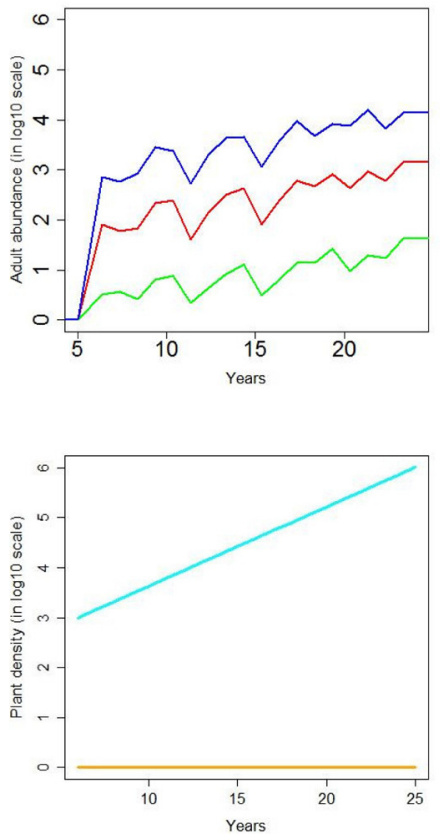

Field
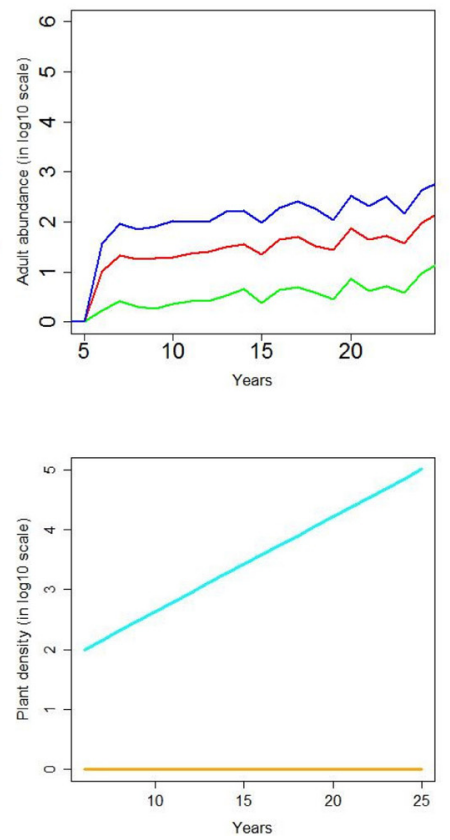

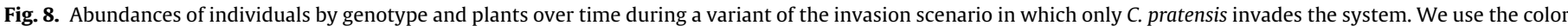

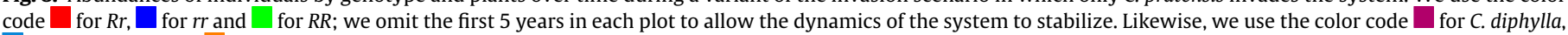
for C. pratensis and for A. petiolata.

though the number of plants (in this case A. petiolata bolting plants) continues increasing.

Moreover, we compared these population trends with others in which we only consider the effect of $C$. pratensis in our system (Fig. 8). In this scenario, we did not model any invasion of $A$. petiolata into the system. In this case, there is no bias in favor of $R R$ and $R r$; the population of all 3 genotypes grows linearly.

Additionally, we simulated a scenario in which the total number of plants in the forest declines exponentially over time from $10^{6}$ to roughly $10^{3}$ in 25 years (Fig. 9). The results show a sustained drop in the population of butterflies in the forest. However, this drop does not translate into an increase in the edge and open field populations. This result could indicate that the number of butterflies that leave the forest because of scarcity of suitable plants and arrive at other habitats is comparatively small, and that these individuals are outnumbered by the local butterflies in the edge and field.

To assess the effect of migration in our system, we repeated our experiments with percentages of emigration of $0.1 \%, 1 \%, 5 \%, 15 \%$ and $20 \%$. We found no significant differences in genotype proportions. However, when this parameter is set to $0 \%$, the genotype proportions in the forest are similar to the rest of the scenarios, while the populations in the edge and the open field collapse at the beginning of the simulations. The forest was most probably the original source of butterflies populating the field at an early stage of the plant invasions.

Mark-recapture data on $1800+P$. oleracea adults in northern Vermont (under conditions similar to the pre-invasion scenario) suggest that these $P$. oleracea in a wooded site were frequently recaptured at a second wooded site approximately $0.75 \mathrm{~km}$ distant (straight-line), but that movements between more distant sites were considerably fewer (of 236 recaptures of $P$. oleracea, 4 moved more than $2 \mathrm{~km}$ (Chew, 1981)).

\subsection{Post-invasion}

Finally, in the post-invasion scenario, the $R R$ genotype is predominant in the forest and edge but not in the field, because this last site is apparently an unsuitable habitat for $A$. petiolata, and thus the ensuing bias favoring $R R$ and $R r$ genotypes is absent (Fig. 10). Therefore, the most abundant genotype in the pre-invasion scenario in the entire system $(r r)$ is still predominant in the field during the post-invasion scenario. The overall number of butterflies in all the populations stabilizes, because the maximum number of plants in each one of the habitats has been already reached. Consequently, the movement dynamics from forest to field stabilize, and the proportion of each phylogenetic configuration is kept with oscillations throughout the simulations. This could be due to migratory influxes, especially from the forest during generations 2 and 3 fleeing from the lack of any good hosts in the forest habitat.

\subsection{Simulation conclusions}

The results qualitatively match our field observations and the experimental laboratory results (Chew et al., 2012; Keeler and Chew, 2008). Moreover, we observe a qualitative match between this model and a previous model assessing population persistence and adaptive allele frequency changes in this species (Morton et al., 2015).

\section{Discussion}

Effects of novel exotic host plants on native herbivorous insects are many (Bezemer et al., 2014) and recent reviews also assess the likelihood of successful colonization of exotic hosts by native insects as a function of spatial proximity between native hosts and non-native plants (Branco et al., 2015), non-native plant phylogenetic and chemical proximity to native hosts (Pearse and Altermatt, 


\section{Generation 1}

Forest
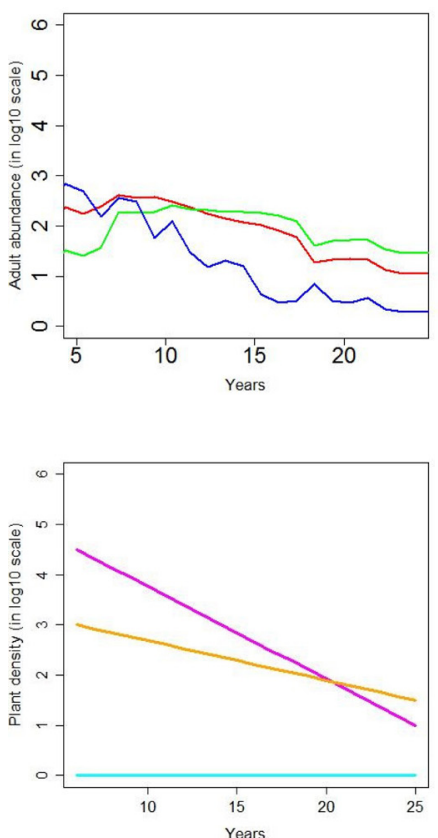

Edge
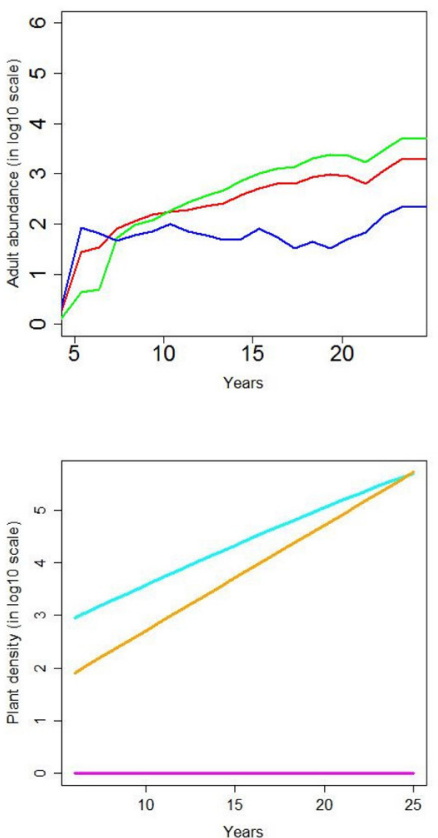

Field
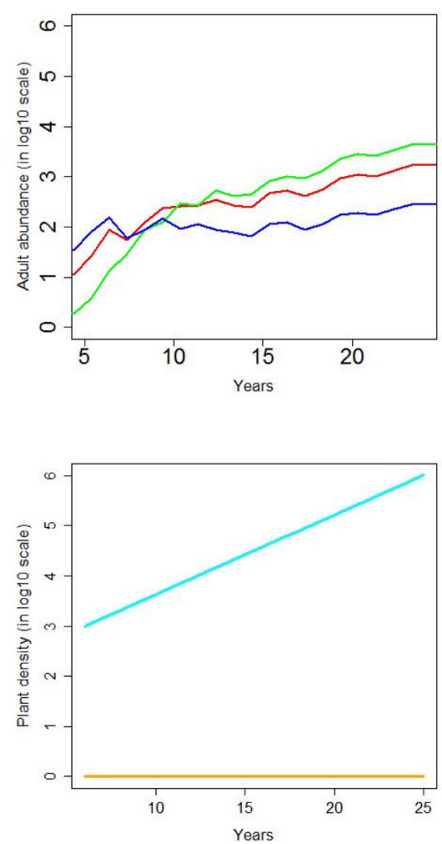

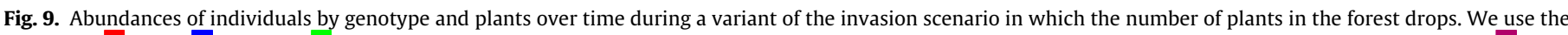

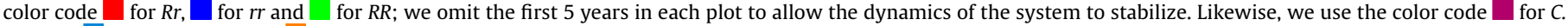
diphylla, for C. pratensis and for A. petiolata.

\section{Generation 1}

Forest

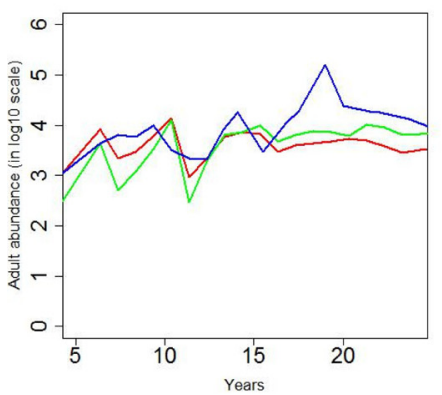

Edge

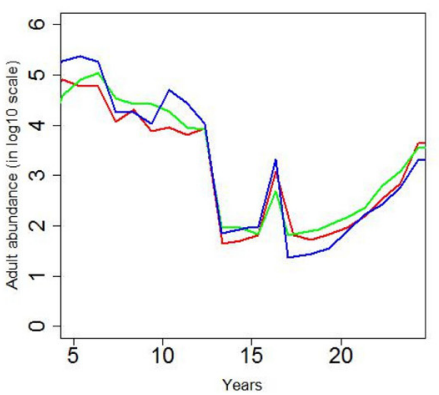

Field

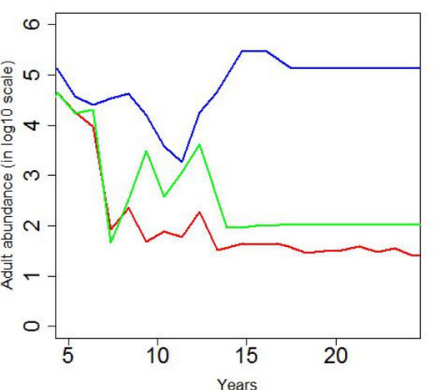

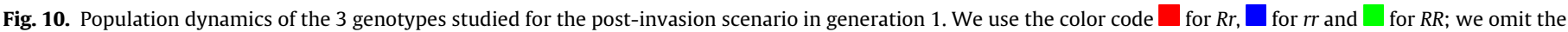
first 5 years in each plot to allow the dynamics of the system to stabilize.

2013), and the geographic range of the native insect species (Jahner et al., 2011). A. petiolata in North America is known to contain novel chemistry (e.g. Cipollini et al., 2005) with deterrent effects on feeding larvae of $P$. oleracea (Haribal et al., 2001). By contrast, C. pratensis is congeneric with a native host of P. oleracea; North American pierid butterflies readily incorporated many other exotic Brassicaceae introduced as weeds or crops (e.g. (Chew, 1981; Shapiro, 1975b)). Ecological analogs of this system elsewhere show parallel biological features including both the contrasting suitabilities of exotic plants encountered recently by native herbivores (Harvey et al., 2010), and adaptation to multiple hosts by butterflies whose several generations per year span changes in plant availability changes in plant availability (Heinen et al., 2016). These contrasting suitabilities of exotic potential hosts may increase population growth and buffer populations when native hosts are limiting (e.g. Shuey et al., 2016) but also contribute to potential popula- tion boom-and-crash dynamics at longer time-scales (Brown et al., 2017).

The inherent complexity of the system modeled here characterizes the current dynamic nature of many ecological systems that is, concurrent, serial, and ongoing invasions of exotic species that include both top-down and bottom-up regulators that have different effects on native species in multi-trophic systems. These regulators can have positive, negative, conflicting, or synergistic effects on native species, and subsequent evolutionary responses, and predicting net effects can be daunting. Our study system has the added complication that the effects and interactions are uneven across space, and across time within a year. To determine the net effects in these complex systems, and to predict a range of potential outcomes, it is necessary to build and simulate models able to capture their spatial and temporal dynamics. 
Our study system aims to capture this complicated and dynamic nature of communities that provide the "ecological theater" in which the "evolutionary play" (Hutchinson, 1965) of native herbivore adaptation occurs. Our first modeling assessment of simpler versions of this system explored the competing roles of "bottomup" (replacement of native host byA. petiolata) and "top-down" (parasitoid predation) processes in the persistence of the native butterfly. Since that assessment, data on the system have made clear the decisive effect of past parasitoid predation (Chew et al., 2012; Herlihy et al., 2014). Our second previous model assessed the possibility of evolutionary escape for P. oleracea through the appearance of predator-free space and evolution to overcome the 'evolutionary trap' (Schlaepfer et al., 2002) created by the invasion of $A$. petiolata (Keeler and Chew, 2008; Morton et al., 2015). This assessment, which did not include consideration of the "good" invasive host, also concluded that residual polymorphism for larval resistance (ability to use A. petiolata) is the most likely source of genetic variation in this evolutionary scenario (Bowden, 1979; Morton et al., 2015). The current modeling system is the most comprehensive, and incorporates additional species as well as space by time interactions. In the following paragraphs, we describe the properties in the system observed from our simulations.

In pre-invasion conditions the wild-type genotype dominates. Alleles for resistance, hypothesized to occur as remnants of a dissected polymorphism (Bowden, 1979), remain because they are selectively neutral in the forested habitat. The phenology of available Brassicaceae host plants causes the butterfly population to shift habitat seasonally, possibly causing seasonal variation in population size (Chew, 1981; Heinen et al., 2016). The conditions modeled show the importance of the population to shift in space as the season progresses, and results in high variance in population size associated with immigration and a high intrinsic rate of increase. High variance in population size is associated with greater extinction risk (Schoener et al., 2003) and may also be associated with reduced effective population size and founder effects or similar stochastic changes in allele frequencies (Crow and Kimura, 1970; Shrimpton and Heath, 2003).

The invasion phase in our model was characterized by rapid proliferation of resistant alleles in $P$. oleracea and rapid increase in overall butterfly abundance. This was caused by both increased host plant abundance, and evolution of resistance (adaptation to the formerly unsuitable host plant $A$. petiolata). $P$. oleracea genotypes adapted to survive on leaves of bolting plants (genotypes $R R$ and $R r$ ) reach large, stable population levels without reducing the population of the genotype unable to adapt to the new host plant $(r r)$. A. petiolata plants remain an evolutionary "opportunity" for $P$. oleracea. The massive pulses in population size shown in the variant of the invasion scenario where $C$. pratensis is not present are associated with large-scale movements of butterflies between habitat types that occur when the carrying capacity of the origin habitat is reached.

In the post-invasion scenario we see genotype sub-structuring in space due to a movement-selection balance because of the habitat-specific distribution of host plants that can be used by all genotypes, and host plants that can be used only by individuals carrying a resistant $R$ allele. As a consequence, the wild genotype ( $r r)$ becomes the least abundant in the forest and edge, which seem to be propitious habitats for $A$. petiolata. On the other hand, the field is an unsuitable environment for $A$. petiolata, and thus $r r$ is still predominant there.

\section{Conclusions}

Evolutionary change can occur rapidly under natural selection if pre-existing genetic variation exists and successful genotypes are able to proliferate. Conditions that present a challenge (Cox, 2004) to population growth would be expected to slow or prevent proliferation of adapted genotypes. We have argued elsewhere (Chew et al., 2012; Keeler et al., 2006; Morton et al., 2015) that top-down population regulation from parasitoid predation on $P$. oleracea larvae might have slowed or prevented evolutionary adaptation in the $P$. oleracea population until that predator was extirpated, thereby releasing predation pressure on the $P$. oleracea population.

Concurrent or closely sequential invasions by exotic potential host plants with contrasting suitabilities for a native herbivore provide a second set of conditions that could favor proliferation of adapted genotypes and incorporation of a formerly poor host into the herbivore's diet. Our contrasting results under the several invasion scenarios suggest that $C$. pratensis, a good exotic host, may have enabled large pulses of butterflies to be exposed to $A$. petiolata, and accelerated the proliferation of resistant genotypes compared to the invasion of $A$. petiolata alone.

In pre-invasion times, $P$. oleracea were normally bivoltine and occasionally facultatively trivoltine (Opler and Krizek, 1984; Opler et al., 1992; Klots, 1951; Scudder, 1889; Chew et al., 2012); the first generation of larvae fed on C. diphylla in forested habitat, and developed into the second generation of adults, which moved into edge and open field habitats where their larval offspring developed on native hosts such as Barbarea orthoceras Ledeb., and exotic, cultivated hosts such as cabbages, Brassica oleracea L., and their relatives. The offspring of the second generation entered diapause as pupae. Under the invasion scenario, our model predicts that because of the extended seasonal availability of suitable host plants, the butterfly should evolve to extend its flight season by adding more generations. This is what has been observed in dietary and voltinism expansions in other butterflies (Herlihy et al., 2014; Shapiro, 1975b; Sims and Shapiro, 1984; Chew et al., 2012). Although early work on F1 hybrids of $P$. oleracea and the related native and non-resistant butterflies $P$. virginiensis and $P$. oleracea (from USA) and $P$. napi $L$. (from Europe) suggested the ability to use $A$. petiolata appears to be transmitted as a Mendelian dominant (Bowden, 1971), more extensive work with $P$. oleracea shows that improved larval survival and faster larval development times are both components of adaptation (Keeler and Chew, 2008), thus suggesting that quantitative genetic variation may also be involved.

We have compared our approach with, to the best of our knowledge, the other existing population dynamics model on the same ecological system. Morton et al. (2015) extended a previous model based on difference equations (Keeler et al., 2006) that captures the evolutionary response of a population of $P$. oleracea to the introduction of $A$. petiolata in its habitat and the replacement of the native host plant $C$. diphylla. However, this model does not take into account the spatial heterogeneity of the system (forest, open field and edge), in contrast to ours. Membrane computing is an ideal framework to model systems that consist of interconnected parts because of the inherently compartmental structure of its models. Likewise, movement dynamics among regions were not captured in the referenced model. We suspect that introducing these dynamics in this model would require revising the entire set of equations to integrate population updates. This is not the case in our model, in which movement dynamics in the metapopulation are modeled as an additional set of rules, not being necessary to modify any previous rule in the system. That is because models in membrane computing are modular, i.e. different aspects of the modeled system can be encapsulated into sets of rules with little interdependence among each other. Moreover, our approach permits us to follow each individual, which paves the way to a deeper analysis of the contributions of individuals to the spatial and temporal dynamics within the metapopulation.

This work is another step on the path to demonstrate the suitability of $\mathrm{P}$ systems as a framework for ecological modeling. 
Their modularity (i.e. simplicity to replace components in a model to accommodate for changes in the modeled system) (RomeroCampero and Pérez-Jiménez, 2008a) and their explicit spatial and temporal dynamics makes them a promising framework in Individual-Based Modeling (Grimm, 1999; Łomnicki, 1999), especially for metapopulation processes in which the spatial dynamics of the system are essential. Our model contributes to the literature of case studies in the application of membrane computing for the spatial modeling of dynamical phenomena in ecology. This series includes models on the habitat of endangered animals (Colomer et al., 2011b,a), along with heuristics for the control of invasive, exotic species (Colomer et al., 2014).

\section{Acknowledgments}

This work was supported by the project TIN2012-37434 of the "Ministerio de Economía y Competitividad" of Spain, co-financed by FEDER funds. Manuel García-Quismondo also acknowledges the support from the National FPU Grant Programme from the Spanish Ministry of Education. Frances S. Chew also acknowledges the support from the National Science Foundation (NSF) grant IBN-9108987. All biological research described here was done in accordance with Massachusetts law, under permit 159.16SCI and its antecedents (to Frances S. Chew).

\section{Appendix A. Supplementary data}

Supplementary data associated with this article can be found, in the online version, at http://dx.doi.org/10.1016/j.ecolmodel.2017. 06.030 .

\section{References}

Alhazov, A., Pérez-Jiménez, M., 2007. Uniform solution of QSAT using polarizationless active membranes. In: Durand-Lose, J., Margenstern, M. (Eds.), Machines, Computations, and Universality. Vol. 4664 of Lecture Notes in Computer Science. Springer, Berlin, Heidelberg, pp. 122-133.

Barbuti, R., Bove, P., Milazzo, P., Pardini, G., 2015. Minimal probabilistic P systems for modelling ecological systems. Theoret. Comput. Sci. 608 (Part 1), 36-56.

Bernardini, F., Gheorghe, M., Krasnogor, N., Muniyandi, R., Pérez-Jiménez, M., Romero-Campero, F., 2006. On P systems as a modelling tool for biological systems. In: Freund, R., Păun, G., Rozenberg, G., Salomaa, A. (Eds.), Membrane Computing. Vol. 3850 of Lecture Notes in Computer Science. Springer, Berlin, Heidelberg, pp. 114-133.

Besozzi, D., Cazzaniga, P., Pescini, D., Mauri, G., 2008. Modelling metapopulations with stochastic membrane systems. Biosystems 91, 499-514.

Bezemer, T.M., Harvey, J.A., Cronin, J.T., 2014. Response of native insect communities to invasive plants. Annu. Rev. Entomol. 59, 119-141.

Bowden, S.R., 1971. American white butterflies Pieridae and English food-plant. J. Lepid. Soc. 25, 6-12.

Bowden, S.R., 1979. Subspecific variation in butterflies: adaptation and dissected polymorphism in Pieris artogeia (Pieridae). J. Lepid. Soc. 33, 77-111.

Branco, M., Brockerhoff, E.G., Castagneyrol, B., Orazio, C., Jactel, H., 2015. Host range expansion of native insects to exotic trees increases with area of introduction and the presence of congeneric native trees. J. Appl. Ecol. 52, 69-77.

Brown, L.M., Breed, G.A., Severns, P.M., Crone, E.E., 2017. Losing a battle but winning the war: moving past preference-performance to understand native herbivore-novel host plant interactions. Oecologia 183, 441-453.

Chew, F., Van Driesche, R., Casagrande, R., 2012. A native butterfly confronts exotic plants and parasitoids. Massachusetts Butterflies 39, 2-7.

Chew, F.S., 1981. Coexistence and local extinction in two pierid butterflies. Am. Nat. $118,655-672$

Cipollini, D., Mbagwu, J., Barto, K., Hillstrom, C., Enright, S., 2005. Expression of constitutive and inducible chemical defenses in native and invasive populations of Alliaria petiolata. J. Chem. Ecol. 31, 1255-1267.

Colomer, M., Margalida, Valencia, A., Palau, L.A., 2014. Application of a computational model for complex fluvial ecosystems: the population dynamics of zebra mussel Dreissena polymorpha as a case study. Ecol. Complex. 20, 116-126.

Colomer, M., Lavín, S., Marco, I., Margalida, A., Pérez-Hurtado, I., Pérez-Jiménez, M. Sanuy, D., Serrano, E., Valencia-Cabrera, L., 2011a. Modeling population growth of Pyrenean chamois (Rupicapra p. pyrenaica) by using P-systems. In: Gheorghe, M., Hinze, T., Paun, G., Rozenberg, G., Salomaa, A. (Eds.), Membrane Computing. Vol. 6501 of Lecture Notes in Computer Science. Springer, Berlin, Heidelberg, pp. 144-159.
Colomer, M.A., Margalida, A., Pérez-Jiménez, M.J., 2013. Population Dynamics P System (PDP) models: a standardized protocol for describing and applying novel bio-inspired computing tools. PLOS ONE 8, e60698.

Colomer, M.A., Margalida, A., Sanuy, D., Pérez-Jiménez, M.J., 2011b. A bio-inspired computing model as a new tool for modeling ecosystems: the avian scavengers as a case study. Ecol. Modell. 222, 33-47.

Colomer-Cugat, M.A., García-Quismondo, M., Macías-Ramos, L.F., Martínez-del Amor, M.A., Pérez-Hurtado, I., Pérez-Jiménez, M.J., Riscos-Núñez, A., Valencia-Cabrera, L., 2014. Membrane System-Based Models for Specifying Dynamical Population Systems. Springer International Publishing, Cham, pp. 97-132 (Chapter 4).

Costantino, R.F., Desharnais, R.A., Cushing, J.M., Dennis, B., 1997. Chaotic dynamics in an insect population. Science $275,389-391$.

Cox, G.W., 2004. Alien Species and Evolution: The Evolutionary Ecology of Exotic Plants, Animals, Microbes, and Interacting Native Species. Island Press, Washington, WA, USA.

Crow, J.F., Kimura, M., 1970. An Introduction to Population Genetics. The Blackburn Press, New York, NY, USA.

Díaz-Pernil, D., Gutiérrez-Naranjo, M., Pérez-Jiménez, M., Riscos-Núñez, A., 2007. Solving subset sum in linear time by using tissue P systems with cell division. In: Mira, J., Álvarez, J. (Eds.), Bio-inspired Modeling of Cognitive Tasks. Vol. 4527 of Lecture Notes in Computer Science. Springer, Berlin, Heidelberg, pp. $170-179$.

Dragomir, C., Ipate, F., Konur, S., Lefticaru, R., Mierla, L., 2014. Model checking kernel P systems. In: Alhazov, A., Cojocaru, S., Gheorghe, M., Rogozhin, Y Rozenberg, G., Salomaa, A. (Eds.), Membrane Computing. Vol. 8340 of Lecture Notes in Computer Science. Springer, Berlin, Heidelberg, pp. 151-172.

Freund, R., Paun, G., Pérez-Jiménez, M.J., 2005. Tissue P systems with channel states. Theoret. Comput. Sci. 330, 101-116.

García-Quismondo, M., 2014. Modelling and Simulation of Real-Life Phenomena in Membrane Computing (Ph.D. thesis). Department of Computer Science and Artificial Intelligence, University of Sevilla.

Garcia-Quismondo, M., 2017. MeCoGUI Webpage. http://www.p-lingua.org/wiki/ index.php/PGSP_systems:_Pieris_oleracea.

García-Quismondo, M., Gutiérrez-Escudero, R., Martínez-del Amor, M.A., Orejuela-Pinedo, E.F., Pérez-Hurtado, I., 2010. P-Lingua 2.0: a software framework for cell-like P systems. Int. J. Comput. Commun. Control 4, 234-243.

García-Quismondo, M., Macías-Ramos, L.F., Pérez-Jiménez, M.J., 2013. Implementing enzymatic numerical P systems for AI applications by means of graphic processing units. In: Topics in Intelligent Engineering and Informatics, vol. 4. Springer, Berlin, Heidelberg, pp. 137-159 (Chapter 10).

García-Quismondo, M., Martínez-del-Amor, M.A., Pérez-Jiménez, M.J., 2015. Probabilistic guarded P systems, a new formal modelling framework. Lect. Notes Comput. Sci. 8961, 194-214.

Gheorghe, M., Ipate, F., 2014. A kernel P systems survey. In: Alhazov, A., Cojocaru, S., Gheorghe, M., Rogozhin, Y., Rozenberg, G., Salomaa, A. (Eds.), Membrane Computing. Vol. 8340 of Lecture Notes in Computer Science. Springer, Berlin, Heidelberg, pp. 1-9.

Gheorghe, M., Ipate, F., Dragomir, C., Mierla, L., Valencia-Cabrera, L., García-Quismondo, M., Pérez-Jiménez, M.J., 2013a. Kernel P systems - version I. In: Proceedings of the Eleventh Brainstorming Week on Membrane Computing (11BWMC). Fénix Editora, Sevilla, España, pp. 97-124.

Gheorghe, M., Ipate, F., Lefticaru, R., Pérez-Jiménez, M.J., Turcanu, A., Valencia Cabrera, L., García-Quismondo, M., Mierla, L., 2013b. 3-Col problem modelling using simple kernel P systems. Int. J. Comput. Math. 90, 816-830.

Grimm, V., 1999. Ten years of individual-based modelling in ecology: what have we learned and what could we learn in the future? Ecol. Modell. 115, 129-148.

Grimm, V., Wyszomirski, T., Aikman, D., Uchmaski, J., 1999. Individual-based modelling and ecological theory: synthesis of a workshop. Ecol. Modell. 115, 275-282.

Gronewold, A., Sonnenschein, M., 1998. Event-based modelling of ecological systems with asynchronous cellular automata. Ecol. Modell. 108, 37-52.

Haribal, M., Yang, Z., Attygalle, A.B., Renwick, J.A.A., Meinwald, J., 2001. A cyanoallyl glucoside from Alliaria petiolata, as a feeding deterrent for larvae of Pieris oleracea. J. Nat. Prod. 64, 440-443.

Harvey, J.A., Biere, A., Fortuna, T., Vet, L.E.M., Engelkes, T., Morriën, E., Gols, R., Verhoeven, K., Vogel, H., Macel, M., Heidel-Fischer, H.M., Schramm, K., van der Putten, W.H., 2010. Ecological fits, mis-fits and lotteries involving insect herbivores on the invasive plant, Bunias orientalis. Biol. Invas. 12, 3045-3059.

Heinen, R., Gols, R., Harvey, J.A., 2016. Black and garlic mustard plants are highly suitable for the development of two native pierid butterflies. Environ. Entomol. 45, 671-676.

Herlihy, M., Van Driesche, R., Wagner, D., 2014. Persistence in Massachusetts of the veined white butterfly due to use of the invasive form of cuckoo flower. Biol. Invas. 16, 2713-2724

Herlihy, M.V., Driesche, R.G.V., Abney, M.R., Brodeur, J., Bryant, A.B., Casagrande, R.A., Delaney, D.A., Elkner, T.E., Fleischer, S.J., Groves, R.L., Gruner, D.S., Harmon, J.P., Heimpel, G.E., Hemady, K., Kuhar, T.P., Maund, C.M., Shelton, A.M., Seaman, A.J., Skinner, M., Weinzierl, R., Yeargan, K.V., Szendrei, Z., 2012. Distribution of Cotesia rubecula (Hymenoptera: Braconidae) and its displacement of Cotesia glomerata in eastern North America. Fl. Entomol 95, 461-467.

Hinze, T., Hayat, S., Lenser, T., Matsumaru, N., Dittrich, P., 2007. Hill kinetics meets P systems: a case study on gene regulatory networks as computing agents in silico and in vivo. In: in: Proceedings of the 8th International Conference on Membrane Computing. Springer-Verlag, Berlin, Heidelberg, pp. 320-335. 
Hutchinson, G.E., 1965. The Ecological Theater and the Evolutionary Play. Yale University Press, New Haven, CT, USA.

Ipate, F., Lefticaru, R., Pérez-Hurtado, I., Pérez-Jiménez, M., Tudose, C., 2012. Formal verification of P systems with active membranes through model checking. In: Gheorghe, M., Păun, G., Rozenberg, G., Salomaa, A., Verlan, S. (Eds.), Membrane Computing. Vol. 7184 of Lecture Notes in Computer Science. Springer, Berlin, Heidelberg, pp. 215-225

Jahner, J.P., Bonilla, M.M., Badik, K.J., Shapiro, A.M., Forister, M.L., 2011. Use of exotic hosts by lepidoptera: widespread species colonize more novel hosts. Evolution 65, 2719-2724.

Jordán, F., Scotti, M., Priami, C., 2011. Process algebra-based computational tools in ecological modelling. Ecol. Complex. 8, 357-363.

Judson, O.P., 1994. The rise of the individual-based model in ecology. Trends Ecol. Evol. 9, 9-14.

Karowe, D., 1990. Predicting host range evolution: colonization of Coronilla varia by Colias philodice (Lepidoptera: Pieridae). Evolution 44, 1637-1647.

Keeler, M.S., Chew, F.S., 2008. Escaping an evolutionary trap: preference and performance of a native insect on an exotic invasive host. Oecologia 156, 559-568.

Keeler, M.S.C., Goodale, B., Reed, B.C.J.M., 2006. Modelling the impacts of two exotic invasive species on a native butterfly: top-down vs. bottom-up effects. J. Anim. Ecol. 75, 777-788.

Klots, A.B., 1951. Field Guide to the Butterflies of North America, East of the Great Plains (Peterson Field Guides). Houghton Mifflin, Boston, MA, USA.

Knerl, A., Bowers, M.D., 2013. Incorporation of an introduced weed into the diet of a native butterfly: consequences for preference, performance and chemical defense. J. Chem. Ecol. 39, 1313-1321.

Łomnicki, A., 1999. Individual-based models and the individual-based approach to population ecology. Ecol. Modell. 115, 191-198.

Macías-Ramos, L.F., Pérez-Jiménez, M.J., Riscos-Núñez, A., Rius-Font, M., Valencia-Cabrera, L., 2013. The efficiency of tissue P systems with cell separation relies on the environment. In: Proceedings of the 13th International Conference on Membrane Computing. Springer-Verlag, Berlin, Heidelberg, pp. $243-256$.

Martinez, M.A., 2013. PMC GPU Webpage. https://sourceforge.net/projects/ pmcgpu/.

Morton, T.A., Thorn, A., Reed, J., Van Driesche, R., Casagrande, R., Chew, F., 2015 Modeling the decline and potential recovery of a native butterfly following serial invasions by exotic species. Biol. Invas. 17, 1683-1695.

Opler, P.A., Krizek, G., 1984. Butterflies East of the Great Plains: An Illustrated Natural History. The Johns Hopkins University Press, Baltimore, MD, USA.

Opler, P.A., Petterson, R.T., Malikul, V., 1992. A Field Guide to Eastern Butterflies. Houghton Mifflin, Boston, MA, USA.

Oracle Corporation, 2017. Java Webpage. http://www.java.com.

Pavel, A., Arsene, O., Buiu, C., 2010. Enzymatic numerical P systems - a new class of membrane computing systems. In: 2010 IEEE Fifth International Conference on Bio-Inspired Computing: Theories and Applications (BIC-TA)., pp. 1331-1336.

Pavel, A.B., Buiu, C., 2012. Using enzymatic numerical P systems for modeling mobile robot controllers. Nat. Comput. 11, 387-393.

Pearse, I.S., Altermatt, F., 2013. Predicting novel trophic interactions in a non-native world. Ecol. Lett. 16, 1088-1094.

Peng, H., Wang, J., Pérez-Jiménez, M.J., 2015a. Optimal multi-level thresholding with membrane computing. Dig. Signal Process. 37, 53-64.
Peng, H., Wang, J., Pérez-Jiménez, M.J., Riscos-Núñez, A., 2014. The framework of P systems applied to solve optimal watermarking problem. Signal Process. 101, 256-265.

Peng, H., Wang, J., Pérez-Jiménez, M.J., Riscos-Núñez, A., 2015b. An unsupervised learning algorithm for membrane computing. Inform. Sci. 304, 80-91.

Peng, H., Wang, J., Pérez-Jiménez, M.J., Wang, H., Shao, J., Wang, T., 2013. Fuzzy reasoning spiking neural P system for fault diagnosis. Inform. Sci. 235, 106-116.

Păun, G., 1999. P systems with active membranes: attacking NP complete problems. J. Autom. Lang. Combin. 6, 75-90.

Păun, G., 2000. Computing with membranes. J. Comput. Sci. 61, 108-143.

Păun, G., Păun, R., 2006. Membrane computing and economics: numerical P systems. Fundam. Inf. 73, 213-227.

Romero-Campero, F.J., Pérez-Jiménez, M.J., 2008a. A model of the quorum sensing system in Vibrio fischeri using P systems. Artif. Life 14, 95-109.

Romero-Campero, F.J., Pérez-Jiménez, M.J., 2008b. Modelling gene expression control using p systems: the Lac Operon, a case study. Biosystems 91, 438-457.

Schlaepfer, M.A., Runge, M.C., Sherman, P.W., 2002. Ecological and evolutionary traps. Trends Ecol. Evol. 17, 474-480.

Schoener, T.W., Clobert, J., Legendre, S., Spiller, D.A., 2003. Life-history models of extinction: a test with island spiders. Am. Nat. 162, 558-573.

Scudder, S.H., 1889. The Buttterflies of the United States and Canada with Special Reference to New England. Houghton Mifflin, Boston, MA, USA

Shapiro, A.M., 1975a. Developmental and phenotypic responses to photoperiod in uni- and bivoltine Pieris napi in California. Trans. R. Entomol. Soc. Lond. 127, 65-71.

Shapiro, A.M., 1975b. The role of watercress (Nasturtium officinaie) as a host of native and introduced pierid butterflies in California. J. Res. Lepid. 14, 158-168.

Shrimpton, J.M., Heath, D.D., 2003. Census vs. effective population size in chinook salmon: large- and small-scale environmental perturbation effects. Mol. Ecol $12,2571-2583$.

Shuey, J., Jacquart, E., Orr, S., Becker, F., Nyberg, A., Littiken, R., Anchor, T., Luchik, D., 2016. Landscape-scale response to local habitat restoration in the regal fritillary butterfly (Speyeria idalia) (Lepidoptera: Nymphalidae). J. Insect Conserv. 20, 773-780.

Sims, S.R., Shapiro, A.M., 1984. Seasonal phenology of Battus philenor (L.) (Papilionidae) in California. J. Lepid. Soc. 37, 281-288.

Song, T., Macías-Ramos, L.F., Pan, L., Pérez-Jiménez, M.J., 2014. Time-free solution to SAT problem using P systems with active membranes. Theoret. Comput. Sci. 529, 61-68.

Valencia-Cabrera, L., García-Quismondo, M., Pérez-Jiménez, M.J., Su, Y., Yu, H., Pan, L., 2013. Modeling logic gene networks by means of probabilistic dynamic P systems. Int. J. Unconv. Comput. 9, 445-464.

Vasile, C., Pavel, A., Dumitrache, I., Păun, G., 2012. On the power of enzymatic numerical P systems. Acta Inform. 49, 395-412.

Wang, T., Zhang, G., Pérez-Jiménez, M.J., Cheng, J., 2015a. Weighted fuzzy reasoning spiking neural P systems: application to fault diagnosis in traction power supply systems of high-speed railways. J. Comput. Theoret. Nanosci. 12 , 1003-1114.

Wang, T., Zhang, G., Zhao, J., He, Z., Wang, J., Pérez-Jiménez, M.J., 2015b. Fault diagnosis of electric power systems based on fuzzy reasoning spiking neural $\mathrm{P}$ systems. IEEE Trans. Power Syst. 3, 1182-1194. 